\title{
- Monazite in Part of the Southern Atlantic Coastal Plain
}

GEOLOGICAL SURVEY BULLETIN 1042-L

This report concerns work done on behalf of the U.S. Atomic Energy Commission and is published with the permission of the Commission

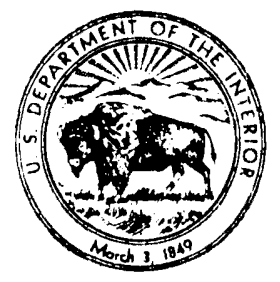




\title{
CONTRIBUTIONS TO ECONOMIC GEOLOGY
}

\section{MONAZITE IN PART OF THE SOUTHERN ATLANTIC COASTAL PLAIN}

\author{
By LINCOLN DRYDEN
}

\section{ABSTRACT}

Sediments and rocks of the inner part of the southern Atlantic Coastal Plain have been sampled and examined for monazite. Most of the samples were collected from the Tuscaloosa formation of Cretaceous age, the McBean and Barnwell formations of Eocene age, and from Pleistocene deposits. A few samples were taken from other formations and from Recent stream and flood-plain sediments. Samples were split, separated in bromoform, and the heavy mineral suites were analyzed for radioactivity with a Geiger counter. The results of these analyses were converted to percentages of monazite in heavy minerals, and these percentages were used to calculate pounds of monazite per cubic yard of sediment.

The 456 samples collected have the following distribution: 293 are from the Tuscaloosa formation, 16 are from the McBean formation, 36 are from the Barnwell formation, and 40 are from Pleistocene deposits. Of the remaining 71 samples collected, 13 are from other deposits of Cretaceous age, 11 from other deposits of Tertiary age, and 47 from Recent deposits. Fewer than one-fourth of the samples (109) contain 0.25 pound or more monazite per cubic yard; the Tuscaloosa, McBean, and Barnwell formations are represented, respectively, by 78,8 , and 6 of these samples. One sample came from the Glendon limestone member of the Byram formation, and 16 from Pleistocene deposits. Only 10 samples contain 1 pound or more monazite per cubic yard. The richest sample has 2.1 pounds per cubic yard. Sampling was done rapidly as reconnaissance and no estimates can be made of the monazite resources, but it is thought that the monazite contents, as found in the samples have considerable lateral and vertical extent.

The monazite in these sediments presumably was derived principally. from the two monazite belts in the Piedmont (Mertie, 1953), but the gea graphic distribution of monazite in sediments along the Coastal Plain does not suggest any particular part of one or both belts as the source, nor does it suggest the way in which monazite was transported and deposited.

All the heavy-mineral suites, except those taken from streams draining the Piedmont province, contain about the same association of minerals. Ilmenite and leucoxene commonly make up half or more of the suite and though percentages of the other minerals are quite variable, an average order of abundance might be: zircon, rutile, monazite, staurolite, kyanite, sillimanite, tourmaline, and spinel. Stream and flood-plain sediments brought from the Piedmont contain these minerals plus epidote, garnet, and hornblende. Streams rising within the Coastal Plain contain the restricted 
suite, and have more monazite in their heavy minerals than do the streams draining the Piedmont.

\section{INTRODUCTION}

The part of the Coastal Plain that extends from New York to Alabama is bounded on the west and northwest along the Fall Line by the higher Piedmont physiographic province and on the east by the Atlantic Ocean. It is generally less than 300 feet in altitude and is characteristically an area of plains and low hills.

The Coastal Plain from North Carolina to Alabama ranges from 100 to 200 miles in width and consists mostly of low, flat, almost featureless plains, but bordering the Fall Line is an inner belt, 10 to 50 miles wide and higher in average altitude than the rest of the Coastal Plain. Stream erosion has progressed much further and local relief in some places reaches a maximum of about 300 feet.

The area covered in this report (fig. 47) is mainly the inner belt. In South Carolina this inner belt has about the same boundaries as the Aiken Plateau, Richland Red Hills, High Hills of Santee, and the Congaree Sand Hills described by Cooke (1936, fig. 1, p. 4, 9-11). In Georgia it corresponds to the Fall Line Hills, Fort Valley Plateau, and the Louisville Plateau of Cooke $(1943$, p. 3). Beyond these States the belt extends without major topographic change to its northeastern end near Wilson, N. C., and southwestward it extends nearly to Wetumpka, Ala. The southwestern part of the area of this report, particularly the part in Alabama, belongs properly to the East Gulf Coastal Plain, but it has furnished comparatively few samples and is here included with the Atlantic Coastal Plain.

Reconnaissance for monazite in sedimentary deposits along the Coastal Plain began in October 1952 and ended in May 1953. Most of this time was spent in a study of Pleistocene shoreline features. Clearly marked topographic features of this kind are generally confined to low altitudes and to the shoreward half or quarter of the Coastal Plain. A short time was spent in the inner half of the Coastal Plain where 60 widely spaced samples were collected and examined in the field. The results of this examination suggested that, apart from monazite deposits associated with shoreline features near the coast, the only other deposits comparatively rich in monazite were to be found in the sediments of the inner belt of the Coastal Plain near the Fall Line. The sediments of this belt were then sampled as extensively as time permitted, and a few samples were taken outside the belt. Sampling was begun in March 1953 and finished in early May 1953. Samples were analyzed for monazite between May and September 1953, and preliminary field determinations of monazite were verified at that time. 


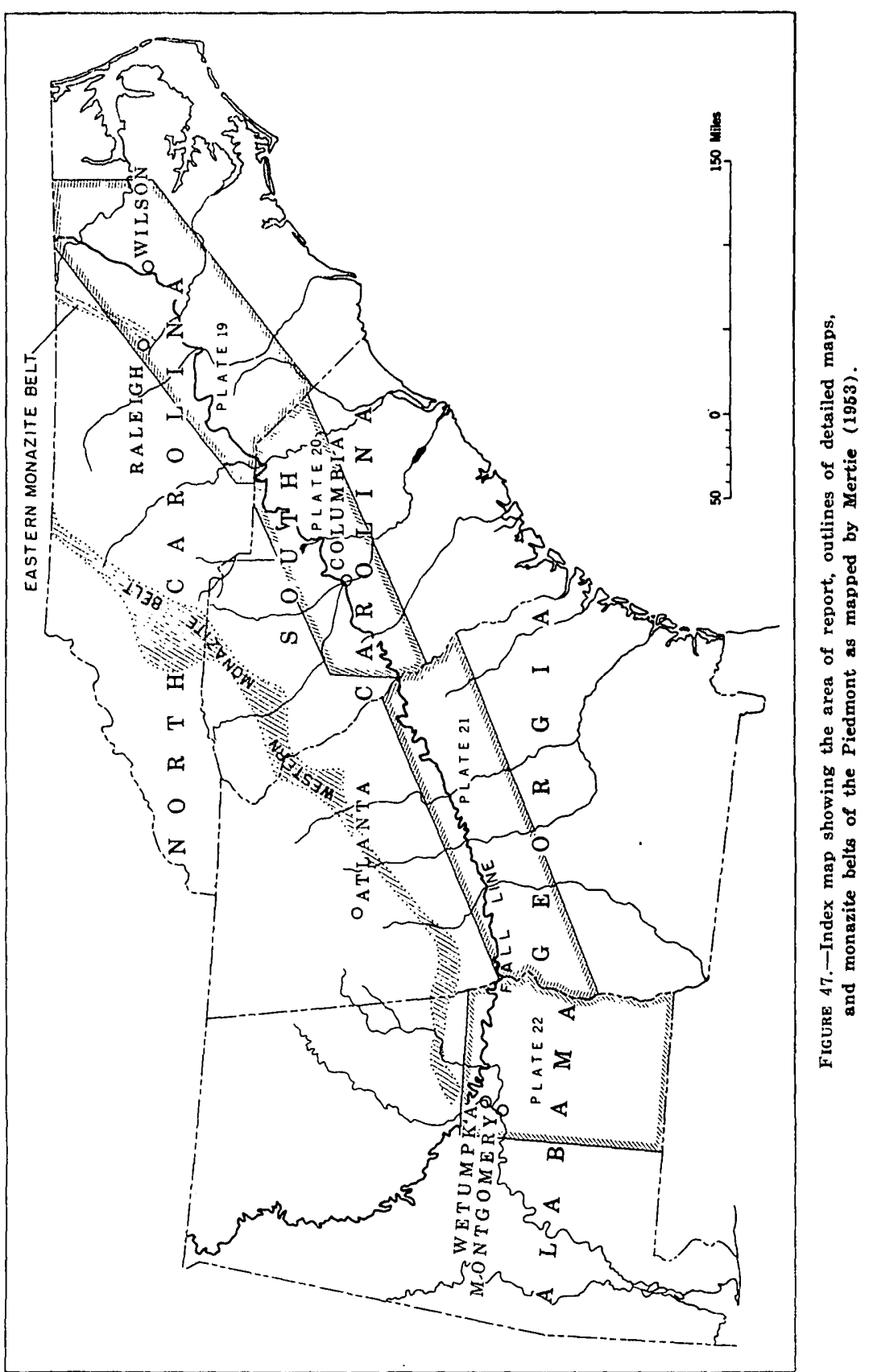


This work was done by the Geological Survey on behalf of the Division of Raw Materials of the U. S. Atomic Energy Commission. The writer was assisted by G. A. Miller who collected most of the samples and helped with the laboratory work. The writer's wife, Clarissa Dryden, was associated with the work throughout and materially assisted in its completion.

\section{GENERAL GEOLOGY}

The geology of the inner belt and of the Coastal Plain as a whole has been described in several reports. Most useful in the present work are those by Stephenson $(1912 ; 1926)$ and Cooke $(1926 ; 1936$; 1943) whose geologic maps were used to identify the formations sampled. A geologic map of the North Carolina Coastal Plain by Berry (1949) also was used.

The topography which characterizes the area is developed principally on rocks of the Tuscaloosa, McBean, and Barnwell formations; to a minor extent it is formed on Pleistocene deposits.

The Tuscaloosa formation of Cretaceous age is the oldest sedimentary formation of the southern Coastal Plain, and it overlies the crystalline rocks of the Piedmont province. It dips gently seaward and passes below successively younger formations of Cretaceous age in that direction. Typically, as in Alabama, the formations of Cretaceous age are succeeded in outcrop shoreward by successively younger formations of Tertiary age, but in parts of South Carolina and Georgia, the McBean and Barnwell formations of Eocene age are widely transgressive. The Barnwell in places overlaps all older Coastal Plain rocks and lies directly on the crystalline rocks of the Piedmont.

Pleistocene deposits are rather widespread in the inner belt in North Carolina and in the northeastern part of South Carolina. Northeast of Wilson, N. C., they are almost undissected, and there mark the northeastern boundary of the inner belt. Southwestward from South Carolina there are fewer and fewer outcrops until Pleistocene sediments occur only in small areas or are absent altogether in the southwestern part of the inner belt.

The character of the Tuscaloosa formation has been described in the reports cited; they stress its extreme variability. Typically, it consists largely of sand that is almost never pure enough to flow readily through the fingers but contains enough silt or clay to make it look and feel mealy. Pebbles, which are common constituents, are in places segregated in well-defined pebble beds but more generally are scattered through finer materials. Lenses of clay, locally sufficiently thick and pure to be commercially valuable, appear to be 
interbedded with coarser sediments. Crossbedding, lensing, and chan-

$\rightarrow$ neling are exposed in almost every outcrop. The Tuscaloosa formation has afforded 293 of the 456 samples collected along the inner half of the Coastal Plain, and of the 293 samples, 76 contain 0.25 pound or more of monazite per cubic yard of rock.

All the other pre-Pleistocene formations are commonly well bedded, contain pebbles or pebble beds only as rare constitutents, and typically

- lack crossbedding, lensing, and channeling. These formations can be distinguished from the Tuscaloosa formation without difficulty.

The McBean formation of Eocene age is made up dominantly of fine- to medium-grained sand and is typically yellowish or greenish. The Barnwell formation of Eocene age is an argillaceous sand which weathers bright red and is more resistant to erosion or slumping than either the Tuscaloosa or McBean formations. Though these two formations of Eocene age are not nearly as extensive in outcrop as the Tuscaloosa formation, they are important in that they contain comparatively high quantities of monazite at many localities. Of the - 16 samples from the McBean formation and 36 from the Barnwell formation, 8 and 6 samples respectively contained 0.25 pound or more of monazite per cubic yard of sediment.

The Pleistocene deposits consists of layers of fairly well-bedded pebbles, sand, and clay. Some of these beds, particularly those containing coarse sand and pebbles, are so much like beds of the Tuscaloosa formation that at places it is difficult or impossible to tell them apart. The writer found this especially true in unmapped outliers on the crystalline rocks of the Piedmont province. Of the 40 samples of Pleistocene sediments studied, 16 contained 0.25 pound or more of monazite per cubic yard of sediment.

Other deposits of Cretaceous age (Black Creek, Peedee, Eutaw, Ripley, and Providence formations) were the source of 13 samples used for radio-activity analysis. Eleven samples were taken from other sediments of Tertiary age (Black Mingo, Wilcox, Clayton, Naheola, Nanafalia, Tuscahoma, Yorktown formations and Glendon limestone member of Byram formation), and 47 specimens came from Recent deposits in streams and flood plains. Table 1 gives the distribution by State and formation. 
TABLE 1.-Distribution of monazite among the heavy minerals and sediments in part of the southern Atlantic Coastal Plain

\begin{tabular}{|c|c|c|c|c|c|c|}
\hline $\begin{array}{c}\text { Sample } \\
\text { No. }\end{array}$ & $\begin{array}{c}\text { Percentage } \\
\text { of sample } \\
<0.5 \mathrm{~mm} \\
\text { in size }\end{array}$ & $\begin{array}{c}\text { Heavy } \\
\text { minerals in } \\
<0.5 \mathrm{~mm} \\
\text { fraction } \\
\text { (percent) }\end{array}$ & $\begin{array}{c}\text { Heavy } \\
\text { minerals } \\
\text { in } \\
\text { sample } \\
\text { (percent) }\end{array}$ & $\begin{array}{c}\text { Monazite } \\
\text { in } \\
\text { heavy } \\
\text { minerals } \\
\text { (percent) }\end{array}$ & $\begin{array}{c}\text { Monazite } \\
\text { in } \\
\text { sample } \\
\text { (percent) }\end{array}$ & $\begin{array}{c}\text { Monazite } \\
\text { per cubic } \\
\text { yard } \\
\text { of sediment } \\
\text { (pounds) }\end{array}$ \\
\hline
\end{tabular}

NORTH CAROLINA

Tuscaloosa formation

\begin{tabular}{|c|c|c|c|c|c|c|}
\hline $7 \mathrm{~B}$ & 40 & 0.9 & 0.4 & 0.3 & 0.0012 & 0.03 \\
\hline $10 \ldots$ & 35 & .75 & .26 & .8 & .0021 & .05 \\
\hline $18 \ldots$ & 85 & 1.5 & 1.3 & .5 & .007 & .18 \\
\hline $19 \ldots$ & 90 & .4 & .36 & .0 & .00 & .00 \\
\hline $20 \ldots$ & 75 & .5 & .38 & .0 & .00 & .00 \\
\hline $21 \ldots \ldots$ & 83 & .3 & .25 & .0 & .00 & .00 \\
\hline 22 & 60 & 1.3 & .78 & .2 & .002 & .05 \\
\hline $23 \ldots$ & 85 & .04 & .03 & .0 & .00 & .00 \\
\hline $24 \ldots$ & 50 & .25 & .13 & .2 & .0003 & .01 \\
\hline $26 \ldots \ldots$ & 93 & .21 & .2 & .4 & .001 & .03 \\
\hline $27 \ldots$ & 45 & .11 & .05 & .0 & .00 & .00 \\
\hline $28 \ldots \ldots$ & 72 & 1.85 & 1.33 & .1 & .002 & .05 \\
\hline $30 \ldots$ & 90 & .08 & .07 & 1.2 & .0008 & .02 \\
\hline $31 \ldots \ldots$ & 65 & 1.1 & .7 & .0 & .00 & .00 \\
\hline $33 \ldots \ldots$ & 95 & .08 & .07 & 3.2 & .002 & .05 \\
\hline $34 \ldots$ & 40 & .4 & .16 & .4 & .00064 & .02 \\
\hline $35 \ldots$ & 59 & .02 & .01 & .0 & .00 & .00 \\
\hline $37 \ldots \ldots$ & 80 & .38 & .3 & .8 & .003 & .08 \\
\hline $38 \ldots \ldots$ & 45 & .53 & .24 & .4 & .001 & .03 \\
\hline $39 \ldots$ & 55 & .31 & .17 & 1.8 & .002 & .05 \\
\hline $41 \ldots \ldots$ & 65 & .18 & .12 & .4 & .0004 & .01 \\
\hline $42 \ldots$ & 94 & .25 & .24 & 1.9 & .0046 & .12 \\
\hline 44 & 90 & .2 & .18 & .6 & .001 & .03 \\
\hline $45 \ldots$ & 80 & .09 & .07 & .8 & .00056 & .01 \\
\hline $46 \ldots \ldots$ & 75 & .16 & .12 & .5 & .0006 & .02 \\
\hline $47 \ldots$ & 50 & 1.1 & .55 & 2.9 & .016 & .4 \\
\hline $48 \ldots \ldots$ & 73 & .4 & .29 & 8.4 & .0244 & .61 \\
\hline $50 \ldots \ldots$ & 60 & .5 & .3 & .7 & .0021 & .05 \\
\hline $51 \ldots$ & 99 & 1.0 & 1.0 & 7.3 & .0725 & 1.81 \\
\hline $52 \ldots$ & 50 & .22 & .11 & 5.9 & .007 & .18 \\
\hline $53 \ldots$ & 30 & .25 & .08 & 1.2 & .0009 & .02 \\
\hline $54 \ldots$ & 65 & .32 & .21 & .5 & .001 & .03 \\
\hline $58 \ldots$ & 65 & .13 & .09 & 1.4 & .001 & .03 \\
\hline $59 \ldots$ & 65 & .35 & .12 & 3.1 & .004 & .1 \\
\hline $60 \ldots$ & 40 & .55 & .22 & 2.7 & .006 & .15 \\
\hline $61 \ldots$ & 65 & .2 & .13 & 2.7 & .004 & .1 \\
\hline $62 \ldots$ & 51 & 1.0 & .51 & 8.8 & .045 & 1.13 \\
\hline $63 \ldots \ldots$ & 75 & .25 & .19 & 4.9 & .009 & .23 \\
\hline $64 \ldots$ & 30 & 2.3 & .70 & 8.9 & .062 & 1.55 \\
\hline $65 \ldots$ & 94 & .29 & .27 & 2.3 & .006 & .15 \\
\hline $66 \ldots \ldots$ & 80 & .2 & .16 & 2.5 & .004 & .1 \\
\hline $67 \ldots \ldots$ & 89 & .06 & .05 & 1.6 & .0008 & .02 \\
\hline
\end{tabular}


MONAZITE IN PART OF THE SOUTHERN ATLANTIC COASTAL PLAIN 399

TABLE 1.-Distribution of monazite among the heavy minerals and sediments in part of the southern Atlantic Coastal Plain-Continued

\begin{tabular}{|c|c|c|c|c|c|c|}
\hline $\begin{array}{c}\text { Sample } \\
\text { No. }\end{array}$ & $\begin{array}{c}\text { Percentage } \\
\text { of sample } \\
<0.5 \mathrm{~mm} \\
\text { in size }\end{array}$ & $\begin{array}{l}\text { Heavy } \\
\text { minerals in } \\
<0.5 \mathrm{~mm} \\
\text { fraction } \\
\text { (percent) }\end{array}$ & $\begin{array}{c}\text { Heavy } \\
\text { minerals } \\
\text { in } \\
\text { sample } \\
\text { (percent) }\end{array}$ & $\begin{array}{c}\text { Monazite } \\
\text { in } \\
\text { heavy } \\
\text { minerals } \\
\text { (percent) }\end{array}$ & $\begin{array}{c}\text { Monazile } \\
\text { in } \\
\text { sample } \\
\text { (percent) }\end{array}$ & $\begin{array}{c}\text { Monazite } \\
\text { per cubic } \\
\text { yard } \\
\text { of sediment } \\
\text { (pounds) }\end{array}$ \\
\hline \multicolumn{7}{|c|}{$\begin{array}{l}\text { NORTH CAROLINA-Continued } \\
\text { Tuscaloosa formation-Continued }\end{array}$} \\
\hline 68. & 45 & 0.23 & 0.1 & 2.6 & 0.003 & 0.08 \\
\hline $69 \mathrm{~A}_{-}$ & 94 & .15 & .14 & .0 & .00 & .00 \\
\hline $70 \ldots$ & 75 & .23 & .17 & 3.3 & .006 & .15 \\
\hline $71 \ldots$ & 45 & .38 & .17 & 1.8 & .0031 & .08 \\
\hline $72 \mathrm{~B}$ & 75 & .4 & .3 & 1.7 & .0051 & .13 \\
\hline 74 & 65 & .24 & .15 & 3.8 & .0057 & .14 \\
\hline 75 & 98 & .17 & .17 & 1.5 & .0025 & .08 \\
\hline $76 \ldots$ & 80 & .52 & .42 & 1.3 & .005 & .13 \\
\hline $77 \ldots$ & 45 & .1 & .05 & 3.7 & .0019 & .05 \\
\hline 79 & 50 & .26 & .13 & 1.9 & .0025 & .06 \\
\hline $80 \ldots$ & 70 & .54 & .38 & 3.3 & .01 & .25 \\
\hline $81 \mathrm{~B}$ & 70 & .8 & .56 & 2.9 & .016 & .4 \\
\hline $83 \ldots$ & 80 & .44 & .35 & 2.2 & .008 & .2 \\
\hline $84 \ldots$ & 85 & .27 & .23 & 1.8 & .004 & .1 \\
\hline $85 \ldots$ & 85 & .38 & .32 & .3 & .0009 & .02 \\
\hline $86 \ldots$ & 70 & .8 & .56 & 1.6 & .009 & .25 \\
\hline 87B. & 75 & .7 & .5 & 3.0 & .015 & .38 \\
\hline $89 \mathrm{~A}$ & 30 & .43 & .13 & 2.9 & .0037 & .09 \\
\hline $90_{\ldots}$ & 50 & .25 & .12 & 2.7 & .003 & .08 \\
\hline $93 \ldots$ & 25 & .37 & .09 & 6.1 & .006 & .15 \\
\hline $94 \ldots$ & 55 & .2 & .07 & 5.4 & .004 & .1 \\
\hline 95 & 35 & .4 & .14 & .0 & .00 & .00 \\
\hline 97. & 70 & .2 & .14 & 3.3 & .005 & .13 \\
\hline 99 & 80 & .28 & .22 & 2.0 & .004 & .1 \\
\hline $100 \mathrm{~A}$ & 40 & .7 & .3 & 1.4 & .0042 & .1 \\
\hline $101 \mathrm{~A}$ & 90 & .27 & .24 & 2.3 & .006 & .15 \\
\hline $102 \mathrm{~A}$ & 65 & .32 & .21 & 6.0 & .013 & .33 \\
\hline $103 \ldots$ & 40 & .16 & .06 & 1.4 & .00084 & .02 \\
\hline $104 \ldots$ & 65 & .7 & .45 & 1.3 & .006 & .15 \\
\hline $105 \ldots$ & 50 & .13 & .07 & 2.1 & .0015 & .04 \\
\hline $106 \mathrm{~A}$ & 75 & .47 & .35 & 3.3 & .012 & .3 \\
\hline $107 \ldots$ & 70 & .1 & .07 & 1.8 & .0013 & .04 \\
\hline $108 \ldots$ & 65 & .4 & .26 & 1.3 & .0034 & .09 \\
\hline $111 \ldots$ & 80 & .2 & .16 & 1.5 & .0024 & .06 \\
\hline $112 B$ & 65 & .36 & .23 & 1.2 & .003 & .08 \\
\hline 113 & 94 & .13 & .12 & 3.0 & .004 & .1 \\
\hline 114 & 70 & .38 & .27 & 2.7 & .007 & .18 \\
\hline $115 \ldots$ & 70 & .34 & .24 & 6.9 & .017 & .43 \\
\hline 116 & 98 & - & - & 2.0 & - & - \\
\hline $117 \ldots$ & 60 & .9 & .54 & 3.4 & .019 & .48 \\
\hline $118 \ldots$ & 88 & .32 & .28 & 0.8 & .002 & .05 \\
\hline $119 \ldots$ & 70 & .27 & .19 & 2.1 & .004 & .1 \\
\hline $120 \ldots$ & 70 & .4 & .28 & 1.1 & .0031 & .08 \\
\hline
\end{tabular}


TABLE 1.-Distribution of monazite among the heavy minerals and sediments in part of the southern Atlantic Coastal Plain-Continued

\begin{tabular}{|c|c|c|c|c|c|c|}
\hline $\begin{array}{c}: \\
\vdots \\
\text { Sample } \\
\text { No: }\end{array}$ & $\begin{array}{c}\text { Percentage } \\
\text { of sample } \\
<0.5 \mathrm{~mm} \\
\text { in size }\end{array}$ & $\begin{array}{c}\text { Heavy } \\
\text { minerals in } \\
<0.5 \mathrm{~mm} \\
\text { fraction } \\
\text { (percent) }\end{array}$ & $\begin{array}{c}\text { Heavy } \\
\text { minerals } \\
\text { in } \\
\text { sample } \\
\text { (percent) }\end{array}$ & $\begin{array}{c}\text { Monazite } \\
\text { in } \\
\text { heavy } \\
\text { minerals } \\
\text { (percent) }\end{array}$ & $\begin{array}{c}\text { Monazite } \\
\text { in } \\
\text { sample } \\
\text { (percent) }\end{array}$ & $\begin{array}{c}\text { Monazite } \\
\text { per cubic } \\
\text { yard } \\
\text { of sediment } \\
\text { (pounds) }\end{array}$ \\
\hline
\end{tabular}

NORTH CAROLINA-Continued

Black Creek formation

\begin{tabular}{r|r|c|c|c|c|c}
\hline 56 & & & & & & \\
110 & 94 & 1.1 & 1.0 & 0.6 & 0.0063 & 0.16 \\
122 & 55 & .12 & .8 & .0 & .00 & .00 \\
& 95 & .6 & .6 & .9 & .0055 & .14 \\
\hline
\end{tabular}

Yorktown formation

\begin{tabular}{r|r|r|r|r|r|r}
\hline $4 \ldots \ldots$ & 35 & 0.74 & 0.26 & 0.1 & 0.0004 & 0.01 \\
$11 \ldots \ldots$ & 70 & .07 & .05 & 2.8 & .0014 & .03 \\
$14 \ldots \ldots$ & .32 & .13 & 2.0 & .0026 & .07 \\
$17 \ldots \ldots$ & 40 & .25 & .1 & 1.2 & .0012 & .03 \\
\hline
\end{tabular}

Pleistocene deposits

\begin{tabular}{|c|c|c|c|c|c|c|}
\hline $43 \ldots$ & 84 & 0.3 & 0.25 & 1.4 & 0.0035 & 0.09 \\
\hline 49 & 70 & .63 & .44 & 1.1 & .0048 & .12 \\
\hline 55 & 80 & .11 & .09 & 3.4 & .0031 & .08 \\
\hline $72 \mathrm{~A}$ & 94 & .5 & .47 & 2.2 & .01 & .25 \\
\hline 73. & 64 & .5 & .32 & 1.5 & .0048 & .12 \\
\hline 78. & 52 & 1.0 & .52 & 4.2 & .022 & .55 \\
\hline 81A. & 70 & .78 & .55 & 2.1 & .0115 & .29 \\
\hline 82. & 42 & 1.0 & .42 & 3.2 & .014 & .35 \\
\hline 87A. & 60 & .5 & .3 & 2.5 & .0075 & .19 \\
\hline 88. & 84 & .62 & .52 & 2.8 & .015 & .38 \\
\hline 89B & 80 & .72 & .58 & 3.0 & .017 & .42 \\
\hline 91. & 50 & .46 & .23 & 0.8 & .002 & .05 \\
\hline 92A & 20 & .36 & .07 & 1.9 & .001 & .03 \\
\hline 92D_ & 35 & .35 & .12 & 0.1 & .0001 & .00 \\
\hline 96. & 65 & .6 & .39 & 6.7 & .026 & .65 \\
\hline 98. & 60. & .8 & .48 & 3.0 & .014 & .35 \\
\hline 101B. & 91 & 1.0 & .91 & 2.9 & .0275 & .68 \\
\hline $102 B$ & 75 & .58 & .43 & 2.9 & .0125 & .31 \\
\hline $106 \mathrm{~B}$ & 78 & .52 & .41 & 2.4 & .01 & .25 \\
\hline 109. & 40 & .29 & .11 & .0 & .00 & .00 \\
\hline $112 \mathrm{~A}$ & 75 & .3 & .23 & .4 & .0009 & .02 \\
\hline 121 & 35 & .4 & .14 & .0 & .00 & .00 \\
\hline
\end{tabular}


MONAZITE IN PART OF THE SOUTHERN ATLANTIC COASTAL PLAIN 401

TABLE 1.-Distribution of monazite among the heavy minerals and sediments in part of the southern Atlantic Coastal. Plain-Continued

\begin{tabular}{|c|c|c|c|c|c|c|}
\hline $\begin{array}{c}\text { Sample. } \\
\text { No. }\end{array}$ & $\begin{array}{c}\text { Percentage } \\
\text { of sample } \\
<0.5 \mathrm{~mm} \\
\text { in size }\end{array}$ & $\begin{array}{c}\text { Heavy } \\
\text { minerals in } \\
<0.5 \mathrm{~mm} \\
\text { fraction } \\
\text { (percent) }\end{array}$ & $\begin{array}{c}\text { Heavy } \\
\text { minerals } \\
\text { in. } \\
\text { sample: } \\
\text { (percent) }\end{array}$ & $\begin{array}{c}\text { Monazile } \\
\text { in } \\
\text { heavy } \\
\text { minerals } \\
\text { (percent). }\end{array}$ & {$\left[\begin{array}{c}\text { Monazite } \\
\text { : in } \\
\text { iample } \\
\text { (percent) }\end{array}\right.$} & $\begin{array}{c}\text { Monazile } \\
\text { per cubic } \\
\text { yard } \\
\text { of sediment } \\
\text { (pounds) }\end{array}$ \\
\hline
\end{tabular}

NORTH CAROLINA-Continued

Stream sediments and miscellaneous samples

\begin{tabular}{|c|c|c|c|c|c|c|}
\hline $1 \ldots$ & - & $\ldots$ & $\ldots$ & 0.2 & $\ldots$ & $\ldots$ \\
\hline $2 \ldots$ & -. & $\ldots$ & $\ldots$ & 1.3 & $\ldots$ & $\ldots$ \\
\hline $3 \ldots$ & - & $\ldots$ & $\therefore$ & .3 & $\ldots$ & $\ldots$ \\
\hline $5 \ldots$ & -- & $\ldots$ & $\ldots$ & .3 & $\ldots$ & $\ldots$ \\
\hline $6 \ldots$ & 92 & 4.95 & 4.55 & .2 & $\ldots$ & $\ldots$ \\
\hline 7A. & -- & $\ldots$. & ... & 1.0 & $\ldots$ & $\ldots$ \\
\hline $8 \ldots$ & $\ldots$ & $\ldots$ & $\ldots$ & 3.6 & $\ldots$ & $-\ldots$ \\
\hline 9 & 89 & 4.0 & 3.6 & .3 & $\ldots$ & $\ldots$ \\
\hline $12 \ldots$ & -- & $-\because-$ & $\ldots$ & 2.1 & $\cdots$ & $\ldots$ \\
\hline $13 \ldots$ & 82 & .33 & .27 & .8 & $\therefore$ & $\ldots$ \\
\hline $15 \ldots$ & -. & $\cdots$ & $\ldots$ & 1.8 & $\ldots$ & $\ldots$ \\
\hline $16 \ldots$ & . . & $\ldots$ & $\therefore$ & 1.0 & $\ldots$ & $\ldots$ \\
\hline 25 & . & $\therefore$ & $\ldots$ & 1.0 & $\ldots$ & $\ldots$ \\
\hline 29 & - & $\ldots$ & $\ldots$ & .2 & $\ldots$ & $\ldots$ \\
\hline 32 & $\therefore$ & $\ldots$ & $\therefore$ & .7 & $\ldots$ & $\ldots$ \\
\hline $36 \ldots$ & 96 & 1.6 & 1.5 & .0 & $\ldots$ & $\ldots$ \\
\hline $40 \ldots$ & 80 & .15 & .12 & .0 & $\ldots$ & $\ldots$ \\
\hline $57 \ldots$ & 40 & 1.2 & .5 & .9 & $\ldots$ & $\ldots$ \\
\hline $69 B_{\ldots}$ & 96 & .2 & $: 2$ & 6.0 & $\ldots$ & $\ldots:$ \\
\hline $92 \mathrm{~B}$ & 69 & 2.95 & 2.0 & 1.5 & $\ldots$ & $\ldots$ \\
\hline $92 \mathrm{C}$ & 77 & 1.9 & 1.47 & .4 & $\therefore$ & $\ldots$ \\
\hline $100 B_{\ldots}$ & 70 & .6 & .4 & 9 & $\ldots$ & $\ldots$ \\
\hline $123 \ldots$ & 98 & .7 & .7 & 2.5 & $\ldots$ & $\ldots$ \\
\hline
\end{tabular}

SOUTH CAROLINA

Tuscaloosa formation

\begin{tabular}{l|l|l|l|l|l|l}
\hline $124 \ldots \ldots$ & 85 & 0.28 & 0.24 & 6.7 & 0.016 & 0.4 \\
$125 \ldots \ldots$ & 87 & .93 & .81 & 6.2 & .05 & 1.25 \\
$126 \ldots \ldots$ & 82 & .43 & .35 & 4.8 & .017 & .43 \\
$127 \ldots \ldots$ & .6 & .27 & 3.1 & .008 & .2 \\
$128 \ldots \ldots$ & 30 & .72 & .22 & 2.0 & .004 & .1 \\
$129 \ldots \ldots$ & .28 & .11 & 1.1 & .001 & .03 \\
$130 \ldots \ldots$ & .63 & .35 & 5.1 & .017 & .43 \\
$131 \ldots \ldots$ & 55 & .19 & .12 & 3.0 & .004 & .1 \\
$132 \ldots \ldots$ & .4 & .28 & 2.8 & .007 & .18 \\
$132 \mathrm{~B} \ldots$ & 53 & .45 & .25 & 3.9 & .01 & .25 \\
$133 \ldots \ldots$ & 50 & .37 & .19 & 2.9 & .006 & .15 \\
$134 \ldots \ldots$ & 78 & .5 & .4 & 2.0 & .008 & .2 \\
$135 \ldots \ldots$ & 35 & .7 & .25 & 2.5 & .0063 & .16 \\
$136 \ldots$ & 80 & .14 & .11 & 7.7 & .008 & $.2 \%$
\end{tabular}

1 Collected from loose, yellow, fine-grained sand thought to be a deposit of Pleistocene age in an area mapped by Cooke $(1936$, pl. 2) as the Tuscaloosa formation of Cretaceous age. 
TABLE 1.-Distribution of monazite among the heavy minerals and sediments in part of the southern Atlantic Coastal Plain-Continued

\begin{tabular}{|c|c|c|c|c|c|c|}
\hline $\begin{array}{c}\text { Sample } \\
\text { No. }\end{array}$ & $\begin{array}{l}\text { Percentage } \\
\text { of sample } \\
<0.5 \mathrm{~mm} \\
\text { in size }\end{array}$ & $\begin{array}{l}\text { Heavy } \\
\text { minerals in } \\
<0.5 \mathrm{~mm} \\
\text { fraction } \\
\text { (percent) }\end{array}$ & $\begin{array}{c}\text { Heavy } \\
\text { minerals } \\
\text { in } \\
\text { sample } \\
\text { (percent) }\end{array}$ & $\begin{array}{c}\text { Monazite } \\
\text { in } \\
\text { heavy } \\
\text { minerals } \\
\text { (percent) }\end{array}$ & $\begin{array}{c}\text { Monazile } \\
\text { in } \\
\text { sample } \\
\text { (percent) }\end{array}$ & $\begin{array}{c}\text { Monazite } \\
\text { per cubic } \\
\text { yard } \\
\text { of sediment } \\
\text { (pounds) }\end{array}$ \\
\hline \multicolumn{7}{|c|}{$\begin{array}{l}\text { SOUTH CAROLINA-Continued } \\
\text { Tuscaloosa formation-Continued }\end{array}$} \\
\hline 137. & 64 & 1.9 & 1.22 & 5.5 & 0.067 & 1.68 \\
\hline 138 & 50 & .7 & .4 & .9 & .004 & .10 \\
\hline 139 & 50 & .21 & .11 & 3.7 & .004 & .1 \\
\hline 140 & 50 & .18 & .09 & 3.4 & .003 & .08 \\
\hline 141 & 85 & .76 & .65 & .7 & .005 & .13 \\
\hline 142 & 75 & .16 & .12 & 1.3 & .0015 & .04 \\
\hline 143 & 35 & .81 & .29 & .2 & .0006 & .02 \\
\hline 144 & 37 & .68 & .25 & 5.7 & .014 & .35 \\
\hline $145 \ldots$ & 65 & .37 & .24 & 4.9 & .012 & .3 \\
\hline $146 \ldots$ & 35 & .43 & .15 & 1.8 & .003 & .08 \\
\hline 148 & 70 & .19 & .13 & 1.3 & .002 & .05 \\
\hline 149 & 94 & .25 & .24 & 2.8 & .0067 & .17 \\
\hline 150 & 94 & .08 & .08 & 2.8 & .002 & .05 \\
\hline 151 & 40 & 2.66 & 1.1 & .6 & .006 & .15 \\
\hline 152 & 55 & .7 & .38 & 2.5 & .009 & .23 \\
\hline 153 & 40 & .27 & .11 & 2.2 & .002 & .05 \\
\hline 154 & 60 & .3 & .18 & 3.0 & .005 & .13 \\
\hline 155 & 71 & .65 & .46 & 5.8 & .027 & .68 \\
\hline 156 & 78 & .66 & .52 & 4.3 & .022 & .55 \\
\hline $157 \ldots$ & 78 & .82 & .64 & 3.9 & .025 & .63 \\
\hline 158 & 50 & .57 & .29 & 1.6 & .005 & .13 \\
\hline 159 & 60 & .5 & .3 & 2.5 & .007 & .18 \\
\hline $160 \ldots$ & 45 & .19 & .09 & 4.7 & .004 & .10 \\
\hline $161 \ldots$ & 55 & .22 & .12 & 2.8 & .004 & .10 \\
\hline $162 \ldots$ & 60 & .4 & .24 & 2.1 & .005 & .13 \\
\hline $163 \ldots$ & 60 & .43 & .26 & .3 & .0007 & .02 \\
\hline $164 \ldots$ & 41 & 1.4 & .58 & 3.4 & .020 & .50 \\
\hline $165 \ldots$ & 80 & .25 & .2 & 1.6 & .003 & .08 \\
\hline $166 \ldots$ & 55 & .28 & .15 & 3.4 & .005 & .13 \\
\hline $168 \ldots$ & 40 & 1.23 & .5 & 1.9 & .01 & .25 \\
\hline 169 & 83 & .5 & .27 & 3.9 & .0105 & .26 \\
\hline $170 \ldots$ & 47 & .9 & .42 & 6.1 & .026 & .65 \\
\hline $171 \ldots$ & 70 & .29 & .2 & .2 & .0005 & .01 \\
\hline $172 \ldots$ & 65 & .28 & .18 & 4.1 & .007 & .18 \\
\hline $173 \ldots$ & 50 & .15 & .08 & 5.8 & .005 & .13 \\
\hline $174 \ldots$ & 50 & .34 & .17 & 2.5 & .004 & .10 \\
\hline $175 \ldots$ & 75 & .42 & .31 & 5.6 & .018 & .45 \\
\hline $176 \ldots$ & 30 & .45 & .14 & .4 & .0006 & .02 \\
\hline $177 \ldots$ & 50 & 1.2 & .6 & 1.5 & .009 & .23 \\
\hline $178 \ldots$ & 85 & .08 & .07 & .6 & .0004 & .01 \\
\hline $179 \ldots$ & 70 & .5 & .35 & .9 & .003 & .08 \\
\hline $180 \ldots$ & 85 & .02 & .02 & .6 & .0001 & .00 \\
\hline $181 \ldots$ & 40 & 2.82 & 1.13 & .9 & .01 & .25 \\
\hline
\end{tabular}




\section{MONAZITE IN PART OF THE SOUTHERN ATLANTIC COASTAL PLAIN 403}

TABLE 1.-Distribution of monazite among the heavy minerals and sediments in part of the southern Atlantic Coastal Plain-Continued

\begin{tabular}{|c|c|c|c|c|c|c|}
\hline $\begin{array}{c}\text { Sample } \\
\text { No. }\end{array}$ & $\begin{array}{l}\text { Percentage } \\
\text { of sample } \\
<0.5 \mathrm{~mm} \\
\text { in size }\end{array}$ & $\begin{array}{c}\text { Heavy } \\
\text { minerals in } \\
<0.5 \mathrm{~mm} \\
\text { fraction } \\
\text { (percent) }\end{array}$ & $\begin{array}{c}\text { Heavy } \\
\text { minerals } \\
\text { in } \\
\text { sample } \\
\text { (percent) }\end{array}$ & $\begin{array}{c}\text { Monazite } \\
\text { in } \\
\text { heavy } \\
\text { minerals } \\
\text { (percent) }\end{array}$ & $\begin{array}{c}\text { Monazile } \\
\text { in } \\
\text { sample } \\
\text { (percent) }\end{array}$ & $\begin{array}{c}\text { Monazite } \\
\text { per cubicic } \\
\text { yard } \\
\text { of sediment } \\
\text { (pounds) }\end{array}$ \\
\hline \multicolumn{7}{|c|}{$\begin{array}{l}\text { SOUTH CAROLINA-Continued } \\
\text { Tuscaloosa formation-Continued }\end{array}$} \\
\hline 184B. & 40 & 0.17 & 0.07 & 3.7 & 0.003 & 0.08 \\
\hline 185 & 90 & .5 & .45 & 4.2 & .019 & .48 \\
\hline $186 \ldots$ & 96 & .11 & .11 & 6.4 & .007 & .18 \\
\hline 187. & 60 & .14 & .08 & 1.3 & .001 & .03 \\
\hline 188 & 34 & .4 & .14 & 1.3 & .002 & .05 \\
\hline 189 & 25 & 1.25 & .31 & 5.5 & .017 & .42 \\
\hline $190 \ldots$ & 65 & .08 & .05 & .1 & .00 & .00 \\
\hline $191 \ldots$ & 75 & .34 & .26 & .6 & .0014 & .04 \\
\hline $192 \ldots$ & 50 & .5 & .25 & 1.3 & .003 & .08 \\
\hline 193B. & 32 & 1.1 & .35 & 4.5 & .016 & .40 \\
\hline $193 \mathrm{C}$ & 64 & .23 & .15 & 1.8 & .003 & .08 \\
\hline $194 \ldots$ & 57 & .5 & .29 & 2.4 & .007 & .18 \\
\hline $196 \ldots$ & 80 & .9 & .72 & 4.1 & .030 & .75 \\
\hline $197 \ldots$ & 72 & .4 & .3 & .0 & .00 & .00 \\
\hline 198 & 85 & .3 & .26 & .4 & .001 & .03 \\
\hline 199B & 90 & .05 & .05 & .0 & .00 & .00 \\
\hline $200 \ldots$ & 94 & .09 & .08 & .4 & .0003 & .01 \\
\hline $202-$ & 50 & .65 & .33 & .4 & .001 & .03 \\
\hline $203 \ldots$ & 65 & .08 & .05 & .0 & .00 & .00 \\
\hline $204 \ldots$ & 50 & .2 & .1 & .4 & .0004 & .01 \\
\hline $205 \ldots$ & 53 & 1.53 & .81 & 2.3 & .019 & .48 \\
\hline 207 & 55 & 1.2 & .66 & 1.4 & .009 & .23 \\
\hline $208 \ldots$ & 45 & .52 & .23 & 4.8 & .011 & .28 \\
\hline 209 & 50 & .28 & .14 & .9 & .001 & .03 \\
\hline $210 \ldots$ & 40 & .94 & .38 & 1.5 & .006 & .15 \\
\hline $211 \ldots$ & 65 & .39 & .25 & 4.8 & .012 & .3 \\
\hline $212 \ldots$ & 43 & .9 & .38 & 5.8 & .022 & .55 \\
\hline $213 \ldots$ & 65 & .34 & .22 & 2.3 & .005 & .13 \\
\hline 214 & 75 & .23 & .18 & 1.3 & .002 & .05 \\
\hline 215 & 100 & .4 & .4 & .7 & .003 & .08 \\
\hline 221 & 79 & .5 & .4 & 3.1 & .012 & .3 \\
\hline 222. & 80 & .23 & .19 & 4.1 & .007 & .18 \\
\hline $223 \ldots$ & 74 & .52 & .39 & 2.5 & .01 & .25 \\
\hline $224 \ldots$ & 70 & .24 & .17 & 2.9 & .005 & .13 \\
\hline $225 \ldots$ & 35 & .57 & .2 & 7.0 & .014 & .35 \\
\hline $226 \ldots$ & 70 & .5 & .35 & .9 & .003 & .08 \\
\hline $227 \ldots$ & 65 & .1 & .065 & 7.7 & .005 & .13 \\
\hline 229 & 79 & 2.3 & 1.8 & 1.5 & .027 & .68 \\
\hline 230B & 50 & .11 & .06 & 2.5 & .002 & .05 \\
\hline $231 \ldots$ & 55 & .27 & .12 & 1.8 & .002 & .05 \\
\hline $232 \ldots$ & 60 & .65 & .39 & 6.9 & .027 & .68 \\
\hline $233 \ldots$ & 75 & .18 & .14 & 1.1 & .002 & .05 \\
\hline 234 & 58 & .72 & .42 & 8.6 & .0364 & .91 \\
\hline
\end{tabular}


TABLE 1.-Distribution of monazite among the heavy minerals and sediments in part of the southern Atlantic Coastal Plain-Continued

\begin{tabular}{|c|c|c|c|c|c|c|}
\hline $\begin{array}{c}\text { Sample } \\
\text { No. }\end{array}$ & $\begin{array}{c}\text { Percentage } \\
\text { of sample } \\
<0.5 \mathrm{~mm} \\
\text { in size. }\end{array}$ & $\begin{array}{l}\text { Hervy } \\
\text { minerals in } \\
<0.5 \mathrm{~mm} \\
\text { fraction } \\
\text { (percent) }\end{array}$ & $\begin{array}{c}\text { Heavy } \\
\text { minerals } \\
\text { in } \\
\text { sample } \\
\text { (percent) }\end{array}$ & $\begin{array}{c}\text { Monazite } \\
\text { in } \\
\text { heavy } \\
\text { minerals } \\
\text { (percent) }\end{array}$ & $\begin{array}{c}\text { Monazile } \\
\text { in } \\
\text { sample } \\
\text { (percent) }\end{array}$ & $\begin{array}{c}\text { Monazite } \\
\text { per cubic } \\
\text { yard } \\
\text { of sediment } \\
\text { (pounds) }\end{array}$ \\
\hline \multicolumn{7}{|c|}{$\begin{array}{l}\text { SOUTH CAROLINA-Continued } \\
\text { Tuscaloosa formation-Continued }\end{array}$} \\
\hline 235 & 65 & 0.77 & 0.50 & 4.2 & 0.021 & 0.53 \\
\hline $236 \ldots$ & 36 & 1.5 & .54 & 6.9 & .037 & .93 \\
\hline $237_{-}$ & 89 & .01 & .01 & 4.5 & .0005 & .01 \\
\hline $238 \ldots$ & 65 & .5 & .33 & 10.1 & .033 & .83 \\
\hline $240 \ldots$ & 35 & .5 & .18 & 2.8 & .005 & .13 \\
\hline $241 \ldots$ & 65 & .8 & .52 & 2.6 & .014 & .33 \\
\hline 242 & 45 & .42 & .19 & 3.1 & .006 & .15 \\
\hline $243 \ldots$ & 40 & 1.79 & .72 & .9 & .007 & .18 \\
\hline $244 \ldots$ & 45 & 1.35 & .61 & .6 & .004 & .1 \\
\hline $245 \ldots$ & 60 & .7 & .42 & .8 & .0032 & .08 \\
\hline $247 \ldots$ & 85 & .24 & .2 & 1.9 & .004 & .1 \\
\hline $248 \mathrm{~A}$ & 80 & .5 & .4 & .0 & .00 & .00 \\
\hline 249 & 89 & .5 & .45 & .2 & .001 & .03 \\
\hline $250 \ldots$ & 67 & .57 & .38 & 5.8 & .022 & .55 \\
\hline 251 & 60. & .13 & .08 & 3.1 & .002 & .05 \\
\hline $252 \ldots$ & 88 & .5 & .44 & 3.5 & .015 & .38 \\
\hline $253 \ldots$ & 80 & .17 & .14 & 4.3 & .006 & .15 \\
\hline $256 \ldots$ & 50 & .3 & .15 & 2.1 & .003 & .08 \\
\hline $257 \ldots$ & 57 & .6 & .34 & 5.2 & .018 & .45 \\
\hline $258 \ldots$ & 55 & .5 & .28 & 5.7 & .016 & .40 \\
\hline $259 \ldots$ & 60 & .4 & .24 & 4.6 & .011 & .28 \\
\hline $260 \ldots$ & 70 & .5 & .35 & 2.7 & .0095 & .23 \\
\hline $262 \ldots$ & 50 & .4 & .2 & 4.2 & .008 & .2 \\
\hline $264 \ldots$ & 55 & .8 & .44 & 2.5 & .011 & .28 \\
\hline $265 \ldots$ & 45 & .6 & .27 & 7.0 & .019 & .48 \\
\hline $266 \ldots$ & 80 & .5 & .40 & 7.0 & .028 & .70 \\
\hline $267 \ldots$ & 40 & .5 & .2 & .0 & .00 & .00 \\
\hline $268 \ldots$ & 84 & 1.8 & 1.5 & .1 & .001 & .03 \\
\hline $269 \ldots$ & 80 & .6 & .48 & 3.6 & .018 & .45 \\
\hline $271 \ldots$ & 33 & .85 & .28 & 9.1 & .026 & .65 \\
\hline $273 \ldots$ & 50 & .19 & .1 & 3.4 & .003 & .08 \\
\hline $276 \ldots$ & 85 & .5 & .43 & 3.6 & .016 & .40 \\
\hline $277 \ldots$ & 82 & 1.1 & .9 & 3.9 & .035 & .88 \\
\hline $280 \ldots$ & 50 & .63 & .32 & 2.5 & .008 & .2 \\
\hline $285 \ldots$ & 65 & 1.43 & .86 & .9 & .007 & .18 \\
\hline $286 \ldots$ & 75 & .33 & .25 & 3.6 & .009 & .23 \\
\hline $287 \ldots$ & 95 & .2 & .2 & .6 & .001 & .03 \\
\hline $291 \ldots$ & 70 & .33 & .23 & 2.5 & .006 & .15 \\
\hline $293 \ldots$ & 70 & .52 & .36 & 3.8 & .014 & .35 \\
\hline $298 \ldots$ & 62 & .5 & .31 & .1 & .0004 & .01 \\
\hline $300 \ldots$ & 30 & .5 & .15 & 4.6 & .007 & .18 \\
\hline $303 \ldots \ldots$ & 40. & .5 & .2 & 8.4 & .017 & .43 \\
\hline $304 A_{\ldots}$ & 94 & .25 & .25 & .0 & .00 & .00 \\
\hline
\end{tabular}


MONAZITE IN PART OF THE SOUTHERN ATLANTIC COASTAL PLAIN 405

TABLE 1.-Distribution of monazite among the heavy minerals and sediments in part of the southern Atlantic Coastal Plain-Continued

\begin{tabular}{|c|c|c|c|c|c|c|}
\hline $\begin{array}{c}\text { Sample } \\
\text { No. }\end{array}$ & $\begin{array}{l}\text { Percentage } \\
\text { of sample } \\
<0.5 \mathrm{~mm} \\
\text { in size }\end{array}$ & $\begin{array}{c}\text { Heavy } \\
\text { minerals in } \\
<0.5 \mathrm{~mm} \\
\text { fraction } \\
\text { (percent) }\end{array}$ & $\begin{array}{c}\text { Heavy } \\
\text { minerals } \\
\text { in } \\
\text { sample } \\
\text { (percent) }\end{array}$ & $\begin{array}{c}\text { Monazite } \\
\text { in } \\
\text { heavy } \\
\text { minerals } \\
\text { (percent) }\end{array}$ & $\begin{array}{c}\text { Monazite } \\
\text { in } \\
\text { sample } \\
\text { (percent) }\end{array}$ & $\begin{array}{c}\text { Monazile } \\
\text { per cubic } \\
\text { yard } \\
\text { of sediment } \\
\text { (pounds) }\end{array}$ \\
\hline \multicolumn{7}{|c|}{$\begin{array}{l}\text { SOUTH CAROLINA-Continued } \\
\text { Tuscaloosa formation-Continued }\end{array}$} \\
\hline $\begin{array}{l}306 \ldots \\
309 \\
312 \ldots \\
314 \mathrm{~A} \\
317 \ldots \\
318 \ldots \\
319 \ldots \\
320 \ldots\end{array}$ & $\begin{array}{l}15 \\
60 \\
25 \\
21 \\
40 \\
50 \\
60 \\
60\end{array}$ & $\begin{array}{l}1.1 \\
.2 \\
.25 \\
.26 \\
.43 \\
.5 \\
.3 \\
.65\end{array}$ & $\begin{array}{l}.17 \\
.12 \\
.06 \\
.055 \\
.17 \\
.25 \\
.18 \\
.39\end{array}$ & $\begin{array}{r}2.1 \\
. .6 \\
3.1 \\
3.4 \\
1.3 \\
2.1 \\
.7 \\
1.7\end{array}$ & $\begin{array}{l}0.0035 \\
.0007 \\
.002 \\
.0017 \\
.002 \\
.005 \\
.001 \\
.007\end{array}$ & $\begin{array}{r}0.09 \\
.02 \\
.05 \\
.04 \\
.05 \\
.13 \\
.03 \\
.18\end{array}$ \\
\hline
\end{tabular}

Black Creek formation

\begin{tabular}{c|c|c|c|c|c|c}
\hline $218 \ldots \ldots$ & 94 & 0.02 & 0.014 & 1.9 & 0.0003 & 0.01 \\
$219 \ldots \ldots$ & 89 & .73 & .65 & .6 & .004 & .10 \\
\hline
\end{tabular}

Peedee formation

\begin{tabular}{l|l|l|l|l|l|l}
\hline $217 \ldots \ldots$ & 78 & 0.47 & 0.36 & 0.0 & 0.00 & 0.00 \\
\hline \multicolumn{5}{c}{ Black Mingo formation }
\end{tabular}

\begin{tabular}{l|l|l|l|l|l|l}
\hline $272 \ldots \ldots$ & 60 & 0.5 & 0.3 & 2.2 & 0.0066 & 0.17 \\
\hline
\end{tabular}

McBean formation

\begin{tabular}{r|r|r|c|c|c|r}
\hline $289 \ldots \ldots$ & 82 & 0.6 & 0.49 & 3.9 & 0.019 & 0.48 \\
$290 \ldots \ldots$ & 45 & .8 & .36 & 9.1 & .033 & .83 \\
$302 \ldots \ldots$ & 56 & 2.3 & 1.3 & 5.3 & .069 & 1.73 \\
$315 \ldots \ldots$ & 40 & .4 & .16 & .5 & .0008 & .02 \\
$316 \ldots \ldots$ & 57 & .5 & .29 & 5.8 & .017 & .43 \\
\hline
\end{tabular}

Barnwell formation

\begin{tabular}{|c|c|c|c|c|c|c|}
\hline $278 \ldots \ldots$ & 60 & 0.8 & 0.48 & 3.9 & 0.019 & 0.48 . \\
\hline $281 \ldots$ & 85 & .13 & .11 & 1.7 & .002 & .05 \\
\hline $282 \ldots \ldots$ & 80 & .1 & .08 & 2.0 & .0016 & .04 \\
\hline $283 \ldots$ & 65 & .07 & .05 & .8 & .0003 & .01 \\
\hline $288 \ldots \ldots$ & 78 & .5 & .39 & 1.4 & .005 & .13 \\
\hline $295 \ldots \ldots$ & 40 & .48 & .2 & 2.7 & .005 & .13 \\
\hline 296A.. & 93 & .27 & .25 & 3.7 & .009 & $.23^{\circ}$ \\
\hline $297 \ldots$ & 80 & .5 & .4 & 1.6 & .0064 & .16 \\
\hline $301 \ldots \ldots$ & 75 & .8 & .62 & 1.8 & .011 & .28 \\
\hline $305 \ldots$ & 55 & .44 & .24 & .8 & .002 & .05 \\
\hline 310 & 40 & .5 & .2 & 5.4 & .011 & .28 \\
\hline 311 & 50 & .4 & .2 & 3.4 & .007 & .18 \\
\hline $314 B_{-}$ & 27 & 3.3 & .9 & 8.3 & .0747 & 1.87 \\
\hline $314 C_{-}$ & 68 & .6 & .41 & 8.1 & .033 & .83 \\
\hline
\end{tabular}


TABLE 1.-Distribution of monazite among the heavy minerals and sediments in part of the southern Atlantic Coastal Plain-Continued

\begin{tabular}{|c|c|c|c|c|c|c|}
\hline$\underset{\substack{\text { So. } \\
\text { Somple }}}{ }$ & $\begin{array}{c}\text { Percentage } \\
\text { of sample } \\
<0.5 \mathrm{~mm} \\
\text { in size }\end{array}$ & $\begin{array}{c}\text { Heavy } \\
\text { minerals in } \\
<0.5 \mathrm{~mm} \\
\text { fraction } \\
\text { (percent) }\end{array}$ & $\begin{array}{c}\text { Heavy } \\
\text { minerals } \\
\text { in } \\
\text { sample } \\
\text { (percent) }\end{array}$ & $\begin{array}{c}\text { Monazite } \\
\text { in } \\
\text { heavy } \\
\text { minerals } \\
\text { (percent) }\end{array}$ & $\begin{array}{c}\text { Monazite } \\
\text { in } \\
\text { sample } \\
\text { (percent) }\end{array}$ & $\begin{array}{c}\text { Monazite } \\
\text { per cubic } \\
\text { yard } \\
\text { of sediment } \\
\text { (pounds) }\end{array}$ \\
\hline
\end{tabular}

SOUTH CAROLINA-Continued

Pleistocene deposits

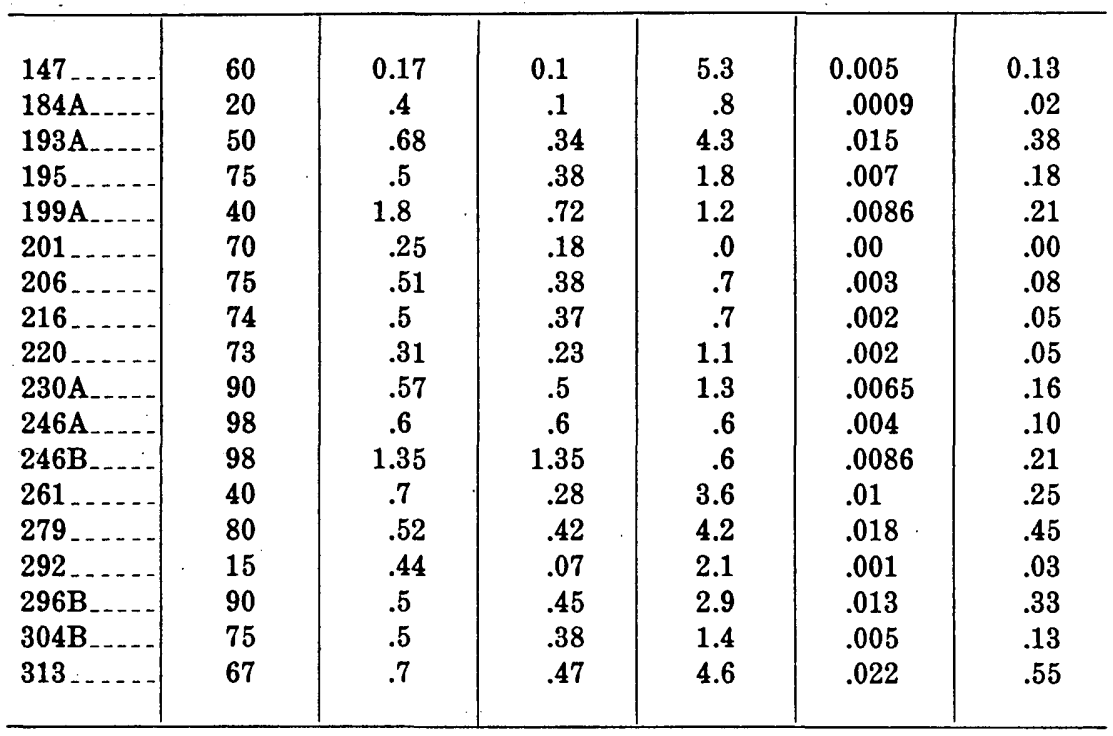

Stream sediments and miscellaneous samples

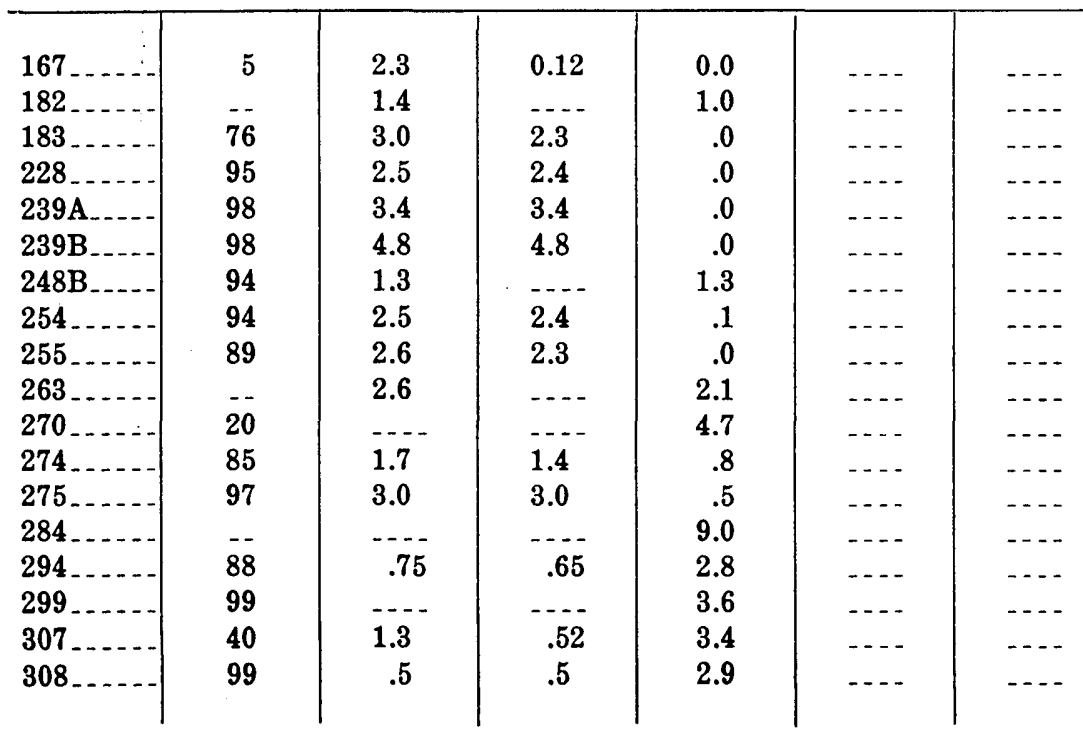


TABLE 1.-Distribution of monazite among the heavy minerals and sediments in part of the southern Atlantic Coastal Plain-Continued

\begin{tabular}{|c|c|c|c|c|c|c|}
\hline $\begin{array}{c}\text { Sample } \\
\text { No. }\end{array}$ & $\begin{array}{c}\text { Percentage } \\
\text { of sample } \\
<0.5 \mathrm{~mm} \\
\text { in size }\end{array}$ & $\begin{array}{c}\text { Heavy } \\
\text { minerals in } \\
<0.5 \mathrm{~mm} \\
\text { fraction } \\
\text { (percent) }\end{array}$ & $\begin{array}{c}\text { Heavy } \\
\text { minerals } \\
\text { in } \\
\text { sample } \\
\text { (percent) }\end{array}$ & $\begin{array}{c}\text { Monazite } \\
\text { in } \\
\text { heavy } \\
\text { minerals } \\
\text { (percent) }\end{array}$ & $\begin{array}{c}\text { Monazile } \\
\text { in } \\
\text { sample } \\
\text { (percent) }\end{array}$ & $\begin{array}{c}\text { Monazile } \\
\text { per cubic } \\
\text { yard } \\
\text { of sediment } \\
\text { (pounds) }\end{array}$ \\
\hline
\end{tabular}

GEORGIA

Tuscaloosa formation

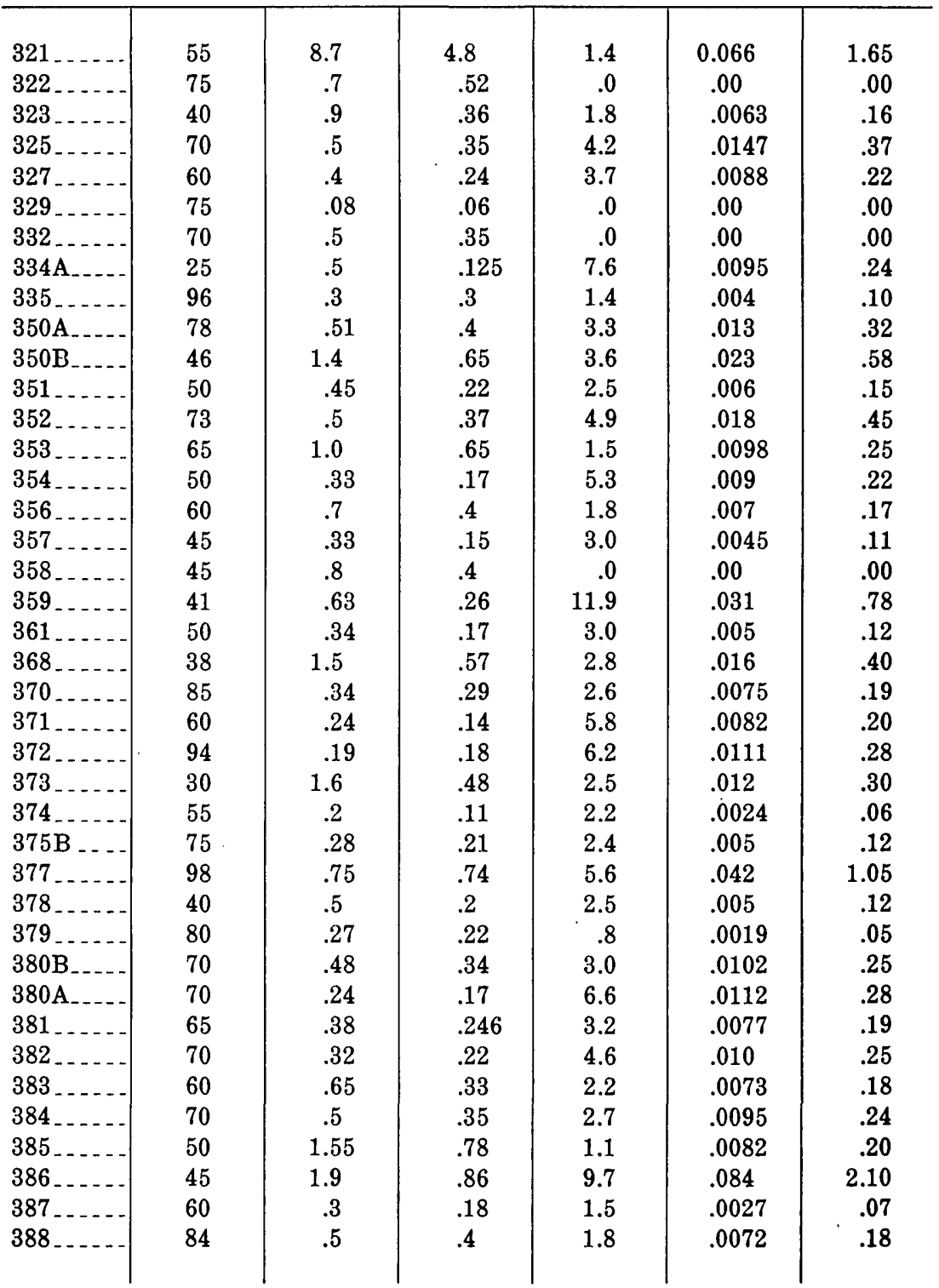


TABLE 1.-Distribution of monazite among the heavy minerals and sediments in part of the southern Atlantic Coastal Plain-Continued

\begin{tabular}{|c|c|c|c|c|c|c|}
\hline $\begin{array}{l}\text { Sample } \\
\text {.. No. }\end{array}$ & $\begin{array}{c}\text { Percentage } \\
\text { of sample } \\
<0.5 \mathrm{~mm} \\
\text { in size }\end{array}$ & $\begin{array}{c}\text { Heavy } \\
\text { minerals in } \\
<0.5 \mathrm{~mm} \\
\text { fraction } \\
\text { (percent) }\end{array}$ & $\begin{array}{c}\text { Heavy } \\
\text { minerals } \\
\text { in } \\
\text { sample } \\
\text { (percent) }\end{array}$ & $\begin{array}{c}\text { Monazite } \\
\text { in } \\
\text { heavy } \\
\text { minerals } \\
\text { (percent) }\end{array}$ & $\begin{array}{c}\text { Monazite } \\
\text { in } \\
\text { sample } \\
\text { (percent) }\end{array}$ & $\begin{array}{l}\text { Monazite } \\
\text { per cubic } \\
\text { yard } \\
\text { of sediment } \\
\text { (pounds) }\end{array}$ \\
\hline
\end{tabular}

GEORGIA-Continued

Wilcox formation

\begin{tabular}{l|l|l|l|l|l|l}
\hline $389 \mathrm{~B}$ & 94 & 0.4 & 0.4 & 0.0 & 0.00 & 0.00 \\
\hline
\end{tabular}

McBean formation

\begin{tabular}{|c|c|c|c|c|c|c|}
\hline $333 \mathrm{~A}$ & 72 & 0.35 & 0.25 & 1.0 & 0.0025 & 0.06 \\
\hline $334 B_{\ldots}$ & 80 & .25 & .2 & 7.4 & .0148 & .37 \\
\hline $337 \mathrm{~A}_{\ldots}$ & 78 & .6 & .46 & 2.0 & .0092 & .23 \\
\hline $337 B_{\ldots}$ & 52 & .4 & .2 & 2.2 & .0044 & .11 \\
\hline $339 \ldots$ & 85 & .5 & .43 & 2.1 & .009 & .22 \\
\hline $341 \ldots$ & 96 & .27 & .26 & .5 & .0013 & .03 \\
\hline 342 & 60 & .48 & .29 & 1.5 & .0044 & .11 \\
\hline $348 B_{-}$ & 96 & .6 & .58 & 2.3 & .013 & .30 \\
\hline $349 \ldots$ & 95 & .5 & .5 & 2.7 & .0135 & .34 \\
\hline 362 & 40 & .58 & .23 & .8 & .002 & .05 \\
\hline 389A_- & 90 & .4 & .36 & 2.8 & .01 & .25 \\
\hline
\end{tabular}

Barnwell formation

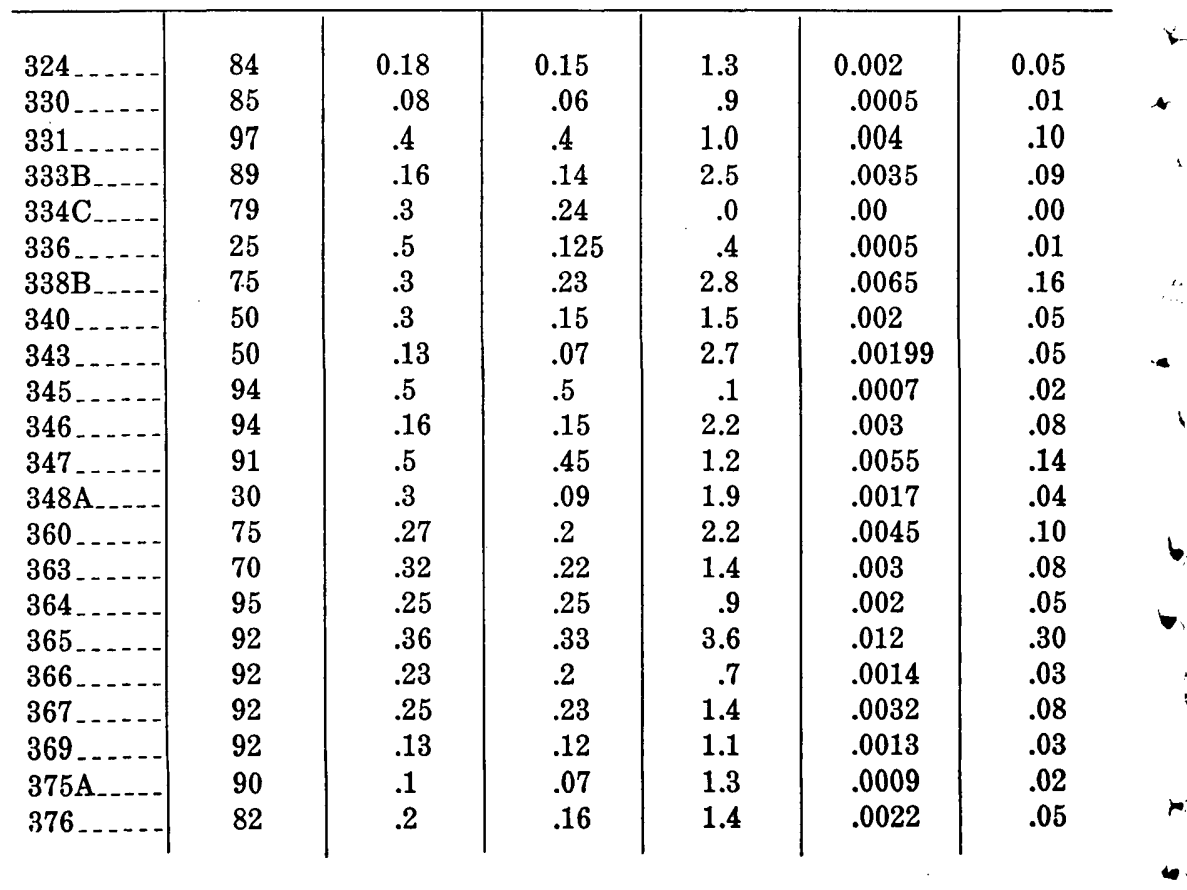


MONAZITE IN PART OF THE SOUTHERN ATLANTIC COASTAL PLAIN 409

TABLE 1.-Distribution of monazite among the heavy minerals and sediments in part of the southern Atlantic Coastal Plain-Continued

\begin{tabular}{|c|c|c|c|c|c|c|}
\hline $\begin{array}{c}\text { Sample } \\
\text { No. }\end{array}$ & $\begin{array}{c}\text { Percentage } \\
\text { of sample } \\
<0.5 \mathrm{~mm} \\
\text { in size }\end{array}$ & $\begin{array}{l}\text { Heavy } \\
\text { minerals in } \\
<0.5 \mathrm{~mm} \\
\text { fraction } \\
\text { (percent) }\end{array}$ & $\begin{array}{c}\text { Heavy } \\
\text { minerals } \\
\text { in } \\
\text { sample } \\
\text { (percent) }\end{array}$ & $\begin{array}{c}\text { Monazite } \\
\text { in } \\
\text { heavy } \\
\text { minerals } \\
\text { (percent) }\end{array}$ & $\begin{array}{c}\text { Monazite } \\
\text { in } \\
\text { sample } \\
\text { (percent) }\end{array}$ & $\begin{array}{c}\text { Monazite } \\
\text { per cubic } \\
\text { yard } \\
\text { of sediment } \\
\text { (pounds) }\end{array}$ \\
\hline
\end{tabular}

GEORGIA-Continued

Stream sediments and miscellaneous samples

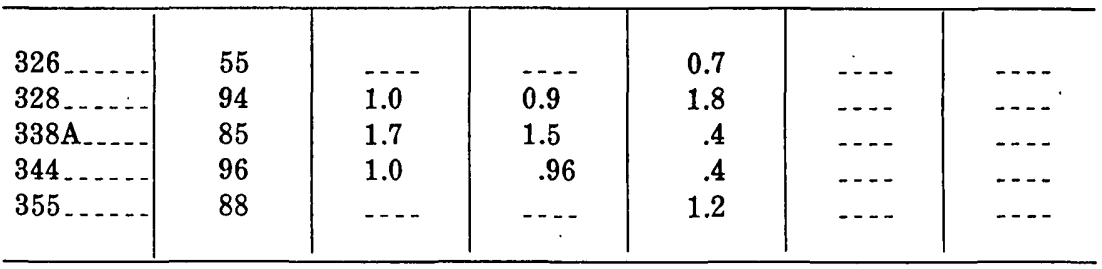

ALABAMA

Tuscaloosa formation

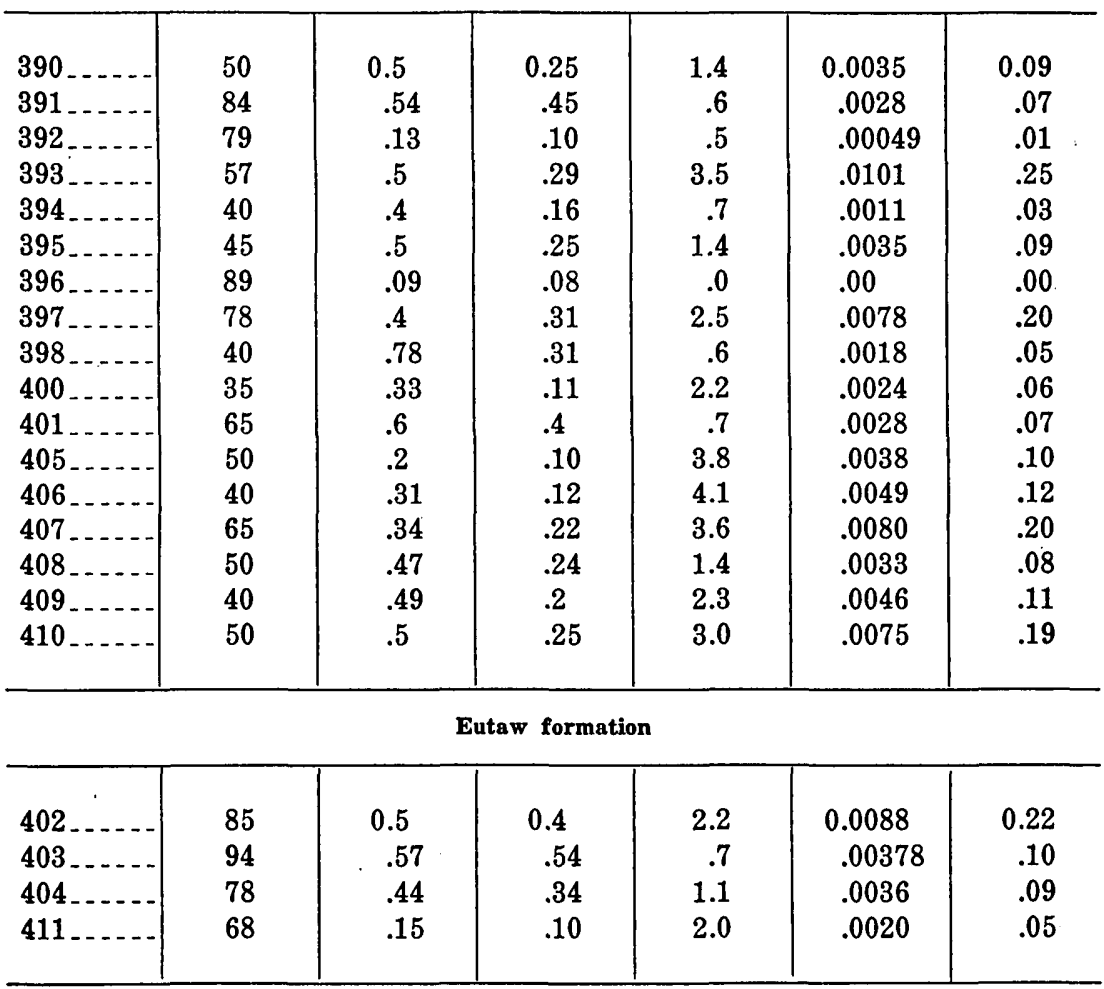

Ripley formation

\begin{tabular}{l|l|l|l|l|l|l}
\hline $412 \ldots .$. & 40 & 0.06 & 0.024 & 2.5 & 0.0006 & 0.01
\end{tabular}


TABLE 1.-Distribution of monazite among the heavy minerals and sediments in part of the southern Atlantic Coastal Plain-Continued

\begin{tabular}{|c|c|c|c|c|c|c|}
\hline $\begin{array}{c}\text { Sample } \\
\text { No. }\end{array}$ & $\begin{array}{c}\text { Percentage } \\
\text { of sample } \\
<0.5 \mathrm{~mm} \\
\text { in size }\end{array}$ & $\begin{array}{l}\text { Heavy } \\
\text { minerals in } \\
<0.5 \mathrm{~mm} \\
\text { fraction } \\
\text { (percent) }\end{array}$ & $\begin{array}{c}\text { Heavy } \\
\text { minerals } \\
\text { in } \\
\text { sample } \\
\text { (percent) }\end{array}$ & $\begin{array}{c}\text { Monazite } \\
\text { in } \\
\text { heavy } \\
\text { minerals } \\
\text { (percent) }\end{array}$ & $\begin{array}{c}\text { Monazite } \\
\text { in } \\
\text { sample } \\
\text { (percent) }\end{array}$ & $\begin{array}{c}\text { Monazite } \\
\text { per cubic } \\
\text { yard } \\
\text { of sedment } \\
\text { (pouinds) }\end{array}$ \\
\hline
\end{tabular}

ALABAMA-Continued

Providence sand

\begin{tabular}{c|c|c|c|c|c|r}
\hline $413 \ldots \ldots$ & 84 & 0.3 & 0.25 & 1.3 & 0.0032 & 0.08 \\
$414 \ldots .$. & 84 & .5 & .4 & 1.5 & .006 & .15 \\
\hline
\end{tabular}

Clayton formation

\begin{tabular}{l|l|l|l|l|l|l}
\hline $416 \ldots \ldots$ & 95 & 0.19 & 0.18 & 1.0 & 0.0018 & 0.05 \\
\hline
\end{tabular}

Naheola formation

\begin{tabular}{l|l|l|l|l|l|l}
\hline $415 \ldots .$. & 90 & 0.5 & 0.45 & 0.0 & 0.00 & 0.00 \\
\hline
\end{tabular}

Nanafalia formation

\begin{tabular}{l|l|l|l|l|l|l}
\hline $417 \ldots \ldots$ & 90 & 0.5 & 0.45 & 1.4 & 0.0063 & 0.16 \\
\hline
\end{tabular}

Tuscahoma sand

\begin{tabular}{l|l|l|l|l|l|l}
\hline $418 \ldots . .$. & 84 & 0.45 & 0.38 & 0.6 & 0.0021 & 0.05 \\
\hline
\end{tabular}

Glendon limestone member of Byram formation

\begin{tabular}{l|l|l|l|l|l|l}
\hline $419 \ldots . .$. & 58 & 1.6 & 0.93 & 1.6 & 0.015 & 0.37 \\
\hline
\end{tabular}

Stream sediments and miscellaneous samples

\begin{tabular}{l|l|l|l|l|l|l}
\hline $399 \ldots \ldots$ & 20 & 0.65 & 0.13 & 0.2 & $\ldots .$. & $\ldots .$. \\
\hline
\end{tabular}




\section{FIELD AND LABORATORY METHODS}

\section{SAMPLING}

Sampling within the inner belt was planned from a general knowledge that some heavy-mineral suites from the Tuscaloosa formation contain significant quantities of monazite, and from field examination of 60 widespread samples. These samples confirmed reports of monazite in the Tuscaloosa formation, but they further showed that other sediments of the inner belt, notably the McBean and Barnwell formations, and Pleistocene deposits, might contain equal or larger amounts of monazite.

Sampling was carried out by reconnaissance methods along public roads and at available exposures. The sediments were identified principally by reference to published small-scale geologic maps. Topographic maps were not available; therefore, the samples lack vertical control. Most samples collected were channel samples and represent about 5 feet of thickness; no sample was taken from less than 2 feet of sediment.

The sampling is not strictly representative of the sediments of the inner belt for several reasons. In some areas low relief results in an absence of natural or artificial exposures; in these areas sampling localities are very widely spaced. The writer believes that low relief is typical of areas underlain by more or less pure sand; as a consequence, pure sand has not been sampled as frequently as its distribution warrants. But in collecting samples from exposures, deliberate effort was made to take material mainly of sand size, and to avoid very fine grained or very coarse grained sediment. It will be shown later (p. 419-420) that monazite is about equally abundant in all sediments sampled that contain material smaller than $0.5 \mathrm{~mm}$ in size.

\section{SPLITTING AND BROMOFORM SEPARATION}

Splitting and bromoform separation of collected samples were used to get a suite of heavy minerals from the minus $0.5 \mathrm{~mm}$ fraction of the sample suitable in size for analysis for radioactivity.

To test a heavy-mineral suite, it is necessary to have a suite weighing at least $0.05 \mathrm{~g}$. To get $0.05 \mathrm{~g}$ of heavy minerals from the minus $0.5 \mathrm{~mm}$ sized fraction, assuming that the heavy-mineral content of this fraction is 0.2 percent, the weight of this fraction has to be at least $25 \mathrm{~g}$. If the original sample is all sand smaller than $0.5 \mathrm{~mm}$, the final split itself should weigh $25 \mathrm{~g}$; if a considerable part of the original sample consists of coarser material, the final split should be such a size that it will contain $25 \mathrm{~g}$ of minus $0.5 \mathrm{~mm}$ material. 
Splitting was done by a Jones 8 - by 10 -inch splitter. The final split was screened through a $0.5 \mathrm{~mm}$ sieve, percentages of plus and minus fractions were estimated, and the material larger than 0.5 $\mathrm{mm}$ was discarded. To verify the estimates, other portions of all samples containing 0.5 pound of monazite or more per cubic yard were split and the minus and plus $0.5 \mathrm{~mm}$ fractions weighed.

These check weighings revealed a systematic error in the direction of too large a percentage reported as minus $0.5 \mathrm{~mm}$, and consequently too great an amount of monazite reported per cubic yard. Of the 53 samples resplit and weighed, 49 gave too high a reading for monazite. The average error was 12.5 percent. For any one sample it was impossible to correct the reported monazite content unless the original sample was resplit, screened, and the minus and plus $0.5 \mathrm{~mm}$ fractions weighed. In table 1 all figures for monazite showing 0.5 pound or more per cubic yard have been corrected by weighing the fractions. The following samples have been corrected in the same way: North Carolina, 102A, South Carolina, 144, 193B, 205, 257, 258, 265, 289, 303, and 316; Georgia, 352 and 368.

The minus $0.5 \mathrm{~mm}$ fraction was weighed, the heavy minerals separated in bromoform and weighed, and the percent of heavy minerals in the fraction calculated. This result when multiplied by the percent of the minus $0.5 \mathrm{~mm}$ fraction gives the percent of heavy minerals in the sample. Occasional checks for total heavy minerals and for monazite made on the discarded plus $0.5 \mathrm{~mm}$ material showed that nearly all of it was practically free of heavy minerals and none of it contained monazite.

\section{DETERMINATION OF MONAZITE CONTENT}

The weight percentage of monazite in a heavy-mineral suite was determined by radioactivity analysis. In this method the heavy-mineral sample requires no special preparation, the only restriction being that its weight must be kept within certain limits. The samples were mounted in stainless steel planchets which were placed in a sample and tube holder. They were then analyzed radiometrically using a $3.5 \mathrm{mg}$ per $\mathrm{cm}^{2}$ end window tube connected to a decimal scaler. To make the results of this type of assay comparable to determinations made by other methods, certain assumptions were made.

The first assumption was that monazite was the one and only source of radioactivity that was measured. This assumption is not strictly true, but the lines of evidence used in establishing the conversion factor discussed below suggest that it can be used as an approximation. More than 100 assayed samples were examined under the microscope, and apart from monazite, no minerals known to be 
radioactive were recognized; about 50 of these samples in which no monazite was seen gave no appreciable radiation.

Second, in converting radiation counts into percentages of monazite and thence into pounds per cubic yard, the assumption was made that all monazite contains an equal quantity of radioactive material per unit weight of mineral. Mertie (1953, p. 12) has shown that this quantity varies rather widely in material from narrowly restricted sources. But Hansen and Cuppels $(1954$, p. $20 ; 1955$, p. 19) and Hansen and White (1954, p. 19) have shown that in large alluvial placers where the distributive province of the stream covers tens of square miles, the composition of the monazite does not vary between such wide limits among samples. This appears to be the result of mechanical mixing of monazite from different sources. It is believed that a wider and more perfect mechanical blending of monazite along ancient stream channels has further reduced the differences between samples, and that though the results of the radioactivity analysis cannot be strictly comparable from sample to sample, they are close enough for estimates of inferred percentages of monazite.

Third, it was assumed that all the radiation from monazite came from thorium or its decay products. But Mertie $(1953$, p. 12) has shown that as much as 12 percent and an average of about 7 percent of the radioactivity of placer monazite is uranium.

Last, it was assumed that no heavy-mineral sample was thick enough to absorb an appreciable amount of the radiation being counted. To test this assumption, 10 samples were analyzed radiometrically. In each case, the same heavy-mineral suite was used, but the weights were successively $0.05 \mathrm{~g}, 0.1 \mathrm{~g}, 0.2 \mathrm{~g}, 0.4 \mathrm{~g}$, and $0.8 \mathrm{~g}$. From $0.05 \mathrm{~g}$ to $0.2 \mathrm{~g}$, radiation counts per unit weight remained about the same but above $0.2 \mathrm{~g}$ the counts per unit weight decreased progressively. For the 10 samples tested, there was no significant absorption of radiation within the sample as long as the weight limit lay between 0.05 to $0.2 \mathrm{~g}$. Since all other heavy-mineral samples were similar in mineral composition (bulk density) and were within these weight limits, it was assumed that no large counting error had been made through absorption within the sample.

In making a radiometric count with a sample containing monazite, the total count is higher than the background; subtracting the background gives the net count. This net count, however, is a function of sample size; dividing net count by the weight of the sample gives the net count per unit weight, which is a value expressing the relative proportion of monazite present. 
Net counts per unit weight are comparable, so that a sample with twice the net count per unit weight of another contains approximately twice as high a percentage of monazite. The conversion factor used in this work to convert net counts per unit weight to percentages was obtained through several methods. One was a weight percentage of monazite (and no other radioactive material) in a heavy-mineral suite furnished by the Brookhaven National Laboratory, Long Island, unit weight of pure monazite and compare these figures with analyses of the sample. Another method was to determine the net counts per unit weight of pure monazite and compare these figures with analyses of suites of known composition. Another was the check of the net counts per unit weight against composition determined by grain counts under the microscope. The different methods all yielded about the same figure: the net count per unit weight multiplied by 0.7 is equal to the percentage of monazite in the heavy-mineral suites from the area studied.

The limits of error in the radiation count of any sample were determined from the percentage of monazite in heavy minerals as given in table 1. For example, sample 58 has 1.4 percent of monazite in heavy minerals, which means 1.4 divided by 0.7 , or net count per unit weight of 2.0. Since the weight of heavy-mineral samples is not given, the error must be calculated on the assumption that any sample weighed between $0.05 \mathrm{~g}$ and $0.2 \mathrm{~g}$. If it weighed $0.05 \mathrm{~g}$, the net count was $2.0 \times 0.05$, or a rate of 0.10 counts per second, or a sample count of 50 in the 500-second interval used for counting each sample or background. A background count of 425 was obtained just before the sample was analyzed, so the total sample and background count would have been 425 plus 50 , or 475 . The error in this count is 4.59 percent, and the rate is 0.95 . To obtain a similar rate for the sample alone, the background value, whose rate is $0.85 \pm 0.04$, is subtracted from $0.95 \pm 0.04$, giving $0.10 \pm \sqrt{(0.0436)^{2}+(0.0412)^{2}}$, or $0.10 \pm 0.06$; the counting error here is so large that the significance of the rate alone may be questioned. But if the sample weight is $0.2 \mathrm{~g}$, the rate for sample and background is $1.25 \pm 0.05$, and the rate for sample alone is $0.40 \pm 0.06$, the percentage error being approximately one-quarter as large as for the $0.05 \mathrm{~g}$ sample. These counting errors directly affect the values of percentage of monazite in heavy minerals, and in the sediment (table 1). In the $0.05 \mathrm{~g}$ heavy-mineral sample, the value of 1.4 percent given in table 1 will vary from 0.56 to 2.24 percent, and the monazite content from 0.01 to 0.05 pound per cubic yard. For the $0.2 \mathrm{~g}$ heavy-mineral sample, corresponding figures are 1.17 to 1.63 percent, and 0.03 to 0.04 pound. 
These figures show that small net counts (or small total counts for 500 seconds) mean large counting errors. Small net counts result from heavy mineral samples of small weight, from low monazite content, or from both. The weight of most heavy mineral samples was kept between $0.05 \mathrm{~g}$ and $0.2 \mathrm{~g}$. However, 62 of the 456 samples assayed weighed less than $0.05 \mathrm{~g}$. They are: North Carolina 11, 17, 33, 55, 67, 69B, 77, 79, 103; South Carolina, 131, 135, 136, 140, $142,147,150,155,173,178,184 \mathrm{~A}, 186,187,197,215,218,227$, $230 \mathrm{~B}, 238,240,245,258,260,262,266,283,288,296,298,300,312$, 315 , 316, 318; Georgia 325, 326, 327, 333A, 335, 338B, 343, 345, 346, 349, 366, 378, 388, 389A ; Alabama 395, 410, 412, 414, 417.

The counting error in analysis of these samples was so large that perhaps they should have been redone from the beginning, so as to obtain a larger heavy-mineral sample for assay. However, it is precisely such samples, with unusually low percentages of heavy minerals, that are of little or no interest in the present work, for even with an average content of monazite in heavy minerals they yield low values of monazite per cubic yard. Large heavy-mineral samples, containing little or no monazite, likewise are subject to large counting errors; these samples, also, are of little interest for the present report, and no attempt has been made to reduce the counting errors in either instance.

For the 109 samples with apparent monazite content of 0.25 pound or more per cubic yard (table 2 ), the heavy minerals contain an average of 4.5 percent monazite. This gives a net count per unit weight of 6.4 , and if we assume for the present calculation an average heavymineral weight of $0.125 \mathrm{~g}$, the average net count would be 0.80 count per second, or 400 counts for the 500 -second interval. The total of sample and background count (assumed to be the same as above) would be 400 plus 425 , or 825 , and the error for this count will be 3.48 percent. The rate for the total count is $1.65 \pm 0.06$ and subtracting the background rate, as before, the rate for sample alone is $0.80 \pm 0.07$. It should be stressed that this value is an average one for the 109 samples, and the errors for this group will be smaller than for the other 347 samples. 
TABLE 2.-Location of samples having an apparent monazite content of 0.25 pound or more per cubic yard of sediment

[All distances were measured by road]

Sample

No.

47

48

51

62

64

$72 \mathrm{~A}$

78

80

$81 \mathrm{~A}, \mathrm{~B}$

82

86

$87 \mathrm{~B}$

88

$89 B$

96

98

$101 B$

(0)

$102 \mathrm{~A}$,

$106 \mathrm{~A}$,

115

117

\section{Location}

\section{NORTH CAROLINA}

Hartnett County; State Route 210, 0.1 mile north of Upper Little River crossing. Harnett County; State Route 87, 0.5 mile north of Olivia.

Moore County; U. S. Route 1, 2.5 miles southwest of Lee County line.

Moore County; U. S. Route 1, near junction with road to Vass.

Harnett County; State Route 210, 0.4 mile north of Cumberland County line.

Moore County; U. S. Route 1, about 2 miles northeast of Aberdeen.

Moore County; U. S. Route 501, 3.5 miles north of Hoke County line.

Moore County; U. S. Route 1, 0.8 miles south of Pinebluff.

Richmond County; U. S. Route $1,1.3$ miles southwest of Hoffman.

Richmond County; U. S. Route 1, 1.6 miles southwest of Moore County line.

Hoke County; U. S. Route 15A, 0.9 mile northeast of Raeford.

Scotland County; U. S. Route 501, 14.6 miles north of junction with U. S. Route 15A north of Laurinburg.

Richmond County; U. S. Route 1, 0.7 mile west of junction with State Route 77 south of Hoffman.

Richmond County; U. S. Route 1, 3.0 miles west of junction with State Route 77 south of Hoffman.

Richmond County; U. S. Route 1, 5.9 miles southwest of junction with State Route 77 south of Hoffman.

Scotland County; U. S. Route 501, 11.1 miles north by junction with U. S. Route $15 \mathrm{~A}$ north of Laurinburg.

Scotland County; U. S. Route 501, 8.0 miles north of junction with U. S. Route $15 \mathrm{~A}$ north of Laurinburg.

Richmond County; U. S. Route 74, 0.5 mile east of junction with N. C. Route 381 east of Hamlet.

Scotland County; U. S. Route 74, 0.7 mile southeast of Richmond County line.

Richmond County; State Route 38, 0.6 mile north of South Carolina line.

Richmond County; State Route 1, 0.4 mile northeast of South Carolina line.

\section{SOUTH CAROLINA}

Mariboro County; State Route 383, 1.1 miles southwest of junction with State Route 79, southwest of Gibson, N. C.

Marlboro County; S. C. Route 38, 0.6 mile south of North Carolina line.

Marlboro County; State Route 77, 1.3 miles south of North Carolina line.

Chesterfield County; U. S. Route 52, 6.5 miles north of junction with U. S. Route 1.

Chesterfield County; State Route 9, 4.1 miles east of Pageland.

Chesterfield County; State Route 102, 1.3 miles southeast of junction with by-pass State Route 9 at Chesterfield.

Marlboro County; State Route 38, 2.6 miles northwest of junction with State Route 383, east of Cheraw.

Chesterfield County; U. S. Route 1, 0.9 mile southwest of junction with U. S. Route 52 south of Cheraw.

Chesterfield County; U. S. Route 1, 4.1 miles south of junction with U. S. Route 52 south of Cheraw.

Chesterfield County; State Route 102, 5.8 miles northwest of Patrick.

Lancaster County; State Route 265, 0.6 mile west-southwest of junction with S. C. Route 903 northeast of Kershaw.

Chesterfield County; State Route 903, 1.4 miles northwest of junction with State Route 151 south of Jefferson.

Chesterfield County; State Route 85, 3.6 miles southwest of junction with State Route 109. 
TABLE 2.-Location of samples having an apparent monazite content of 0.25 pound or more per cubic yard of sediment-Continued

Sample

No.

\section{SOUTH CAROLINA-Continued}

170

175

181

185

189

193A,

Chesterfield County; State Route 102, 4.0 miles northwest of Patrick.

Chesterfield County; U. S. Route 52, 4.7 miles south of junction with U. S. Route 1 at Cheraw.

Marlboro County; State Route 9, 2.1 miles east-southeast of junction with U. S. Route 15 near Bennettsville.

Chesterfield County; S. C. Route 102, 0.3 mile south of Patrick.

Chesterfield County; U. S. Route 1, 3.4 miles northeast of junction with State Route 85 near McBee.

Chesterfield County; State Route 151, 0.7 mile northwest of McBee.

Chesterfield County; State Route 102, 4.4 miles south of Patrick.

Darlington County; State Route 102, 3.8 miles south of Chesterfield County line.

Chesterfield County; U. S. Route 1, 2.0 miles south of McBee.

Kershaw County; U. S. Route 1, 2.4 miles southwest of Cassatt.

Kershaw County; U. S. Route 1, 5.1 miles southwest of Bethune.

Lee County; State Route 341, 3.0 miles southeast of Kershaw County line.

Kershaw County; U. S. Route 1, 3.9 miles northeast of junction with State Route 34 east of Camden.

Kershaw County; U. S. Route 601, 8.4 miles south of Westville.

Kershaw County; U. S. Route 521, 0.9 mile south of Camden.

Lee County; State Route 34, 6.3 miles east of Kershaw County line.

Kershaw County; State Route 34, 6.8 miles east-southeast from junction with $U$. S. Route 1 east of Camden.

Kershaw County; State Route 34, 3.9 miles east-southeast from junction with U. S. Route 1 east of Camden.

Kershaw County; U. S. Route 6011.4 miles south of junction with U. S. Route 1 west of Camden.

Kershaw County; U. S. Route 1, 1.7 miles northeast of Blaney.

Richland County; U. S. Route 1, 0.4 mile southwest of Pontiac.

Kershaw County; U. S. Route 601, 6.7 miles south of junction with State Rioute 12.

Richland County; U. S. Route 1, 1.7 miles northeast of Dentsville.

Richland County; U. S. Route $601,3.0$ miles north of junction with U. S. Route 76.

Richland County; State Route 262, 4.8 miles west of junction with U. S. Route 601 .

Richland County; State Route 262, 12.4 miles west of junction with U. S. Route 601 .

Richland County; near University of South Carolina, Columbia.

Lexington County; U. S. Route 1, 14.4 miles east of junction with State Route 245 near Leesville.

Lexington County: State Route 215, 7.1 miles northeast of junction with State Route 6 at Edmund.

Richland County; Quarry of Southeastern Sand Company, off U. S. Route 321, about 6 miles by road from junction with State Route 215 southwest of Columbia.

Richland County; U. S. Route 76, 12.2 miles east of junction with U. S. Route 1 at Columbia.

Richland County; U. S. Route 76, 6.9 miles east of Lykesland.

Lexington County; U. S. Route 321, 2.7 miles south of junction with U. S. Route 21 south of Columbia.

Lexington County; State Route 215, 2.8 miles northeast of junction with State Route 6 at Edmund.

Lexington County; State Routes 6 and 215, 0.4 mile south of their junction at Edmund.

Lexington County; U. S. Route 178, 6.6 miles southeast of junction with State Route 391 east of Batesburg. 
TABLE 2.-Location of samples having an apparent monazite content of 0.25 pound or more per cubic yard of sediment-Continued

Sample

No.

289

290

293

296B

301

302

303

310

313

314B, C Aiken County; Scott-Seivern Rioad, at crossing of the South Fork of the Edisto River.

350A, B Baldwin County; State Route 24, 7.9 miles southeast of junction with State Route 22 east of Milledgeville.

\section{Location}

SOUTH CAROLINA-Continued

Lexington County; U. S. Route 321, 2.7 miles south of the Gaston fire tower.

Calhoun County; Near U. S. Route 21, 0.05 mile south of Beaver Creek, about 20 miles south-southeast of Columbia.

Aiken County; State Route 391, 0.6 mile north of junction with State Route 39 south of Leesville.

Aiken County; State Route 19, 3.5 miles southeast of Edgefield County line.

Aiken County; State Route 39, 2.3 miles northwest of junction with the ScottSeivern road, near Wagener.

Lexington County; Junction of U. S. Route 321 and State Route 3, 1.1 miles south of Swansea.

Aiken County; U. S. Route 1, 0.6 mile north of Shaw's Creek, about 4 miles north of Aiken.

Aiken County; State Route 215, 2.8 miles east of junction with U. S. Route 78 east of Aiken.

Aiken County; Scott-Seivern Road, 0.9 mile southwest of the South Fork of the Edisto River.

Orangeburg County; U. S. Route 321, 1.1 miles south of North, S. C.

\section{GEORGIA}

McDuffie County; State Route 12, 4.5 miles west of Thomson.

McDuffie County; State Route 17, 9.8 miles north of Wrens.

Jefferson County; Reedy Creek, about 2 miles northeast of Mathews.

Washington County; State Route 24, 2.8 miles west of Sandersville.

Washington County; State Route 24, 3.1 miles west of Sandersville, at creek crossing.

Baldwin County; State Route 24, 4.2 miles southeast of junction with State Route 22 east of Milledgeville.

Baldwin County; about 4 miles southwest of Hardwick.

Bibb County; State Route 49, 5.8 miles northeast of Macon.

Jefferson County; State Route 78, about 1 mile northeast of Wadley, at creek crossing.

Wilkinson County; State Route 18, 0.6 mile north of junction with Ga. Route 57. Bibb County; U. S. Route $80,2.3$ miles east of Lizella.

Crawford County; State Route 128, at Flint River crossing southwest of Roberta.

Taylor County; State Route 137, 4.5 miles northeast of Butler.

Talbot County; State Route 96, 2.8 miles east of Junction City.

Talbot County; at junction of U. S. Route 80 and State Route 41 west of Geneva.

Muscogee County, U. S. Route 80, 1.1 miles northeast of junction with road to Flat Rock.

Randolph County; State Route 266, 4.5 miles southwest of Cuthbert.

\section{ALABAMA}

Elmore County; State Route 14, 5.4 miles east of junction with State Route 11 at Wetumpka.

Houston County; U. S. Route 231, 11.2 miles north of the Florida State line. 


\section{DISTRIBUTION OF MONAZITE}

The localities sampled are shown on parts of four State maps (pls. 19, 20, 21, and 22). Localities 1-123 are in North Carolina, 124-320 in South Carolina, 321-389 in Georgia, and 390-419 in Alabama. If more than one sample was taken from the same locality, each sample has an A, B, or C added to the number. From a total of 419 localities, 456 samples have been collected and analyzed.

For most sample localities shown on the plates 19, 20, 21, and 22, three numbers are given. The top one is the sample number. The middle number is the percentage of monazite in heavy minerals, and the bottom number is pounds of monazite per cubic yard of sediment. Poundage was not calculated for a group of nonrepresentative samples consisting of natural concentrates, selected parts of sediment rich in heavy minerals, waste washings from quarries, and heavy-mineral streaks in gutters. Monazite contents were calculated by using 2,500 pounds as the weight of a cubic yard of sediment in place.

\section{RELATION TO GRAIN SIZE OF SEDIMENTS}

Relations among grain size, percents of heavy minerals, and percents of monazite in heavy minerals and in sediments are given in table 3 for 151 samples from the Tuscaloosa formation in South Carolina. Percents of heavy minerals in the minus $0.5 \mathrm{~mm}$ fraction increase on the average with an increase in the grain size of the sediment. For example, the top nine numbers in column 3 (68 samples) average 0.70 percent of heavy minerals in the minus $0.5 \mathrm{~mm}$ fraction, whereas the bottom nine (83 samples) average 0.41 percent. The percentage of monazite in the heavy minerals is also somewhat greater in samples of coarser grain size: the top nine percentages average 3.34 and the bottom nine, 2.58 percent. But the

; percentages of monazite in the sediments (column 5, which is the product in each case of columns 1,3 , and 4) average 0.0078 percent for the top nine and 0.0081 percent for the bottom nine. In column 5 , if values for the two coarsest and the two finest grained samples are disregarded, there would seem to be little correlation between the grain size of the sample and the percentage of monazite in it. But because the monazite grains in the inner belt of the Coastal Plain are smaller than $0.5 \mathrm{~mm}$, very coarse grained sediments in this belt that have no grains smaller than $0.5 \mathrm{~mm}$ will contain no monazite.

The grain size and monazite content of the Tuscaloosa and other formations within the inner belt differ from those of placers in Pleistocene deposits nearer the Atlantic coast. The sand in these placers 
is probably all smaller than $0.5 \mathrm{~mm}$ in grain size with the exception of some of the sand at Trail Ridge, Clay County, Fla. Monazite grains from the Pleistocene sediments are small: 95 percent of them from one placer sample pass through a $0.125 \mathrm{~mm}$ sieve. Grains of monazite from the Tuscaloosa formation are larger than this. No study of their size distribution was made, but it has been observed that many of them are in the $0.25-0.5 \mathrm{~mm}$ range. The sand of the Pleistocene deposits, unlike that of the Tuscaloosa formation, is well sorted, and appears to have been worked and reworked repeatedly. It may be that abrasion during this process diminished the size of the monazite grains; this wear and ensuing destruction may also account for the fact that monazite is less common in the Pleistocene deposits than in the Tuscaloosa, McBean, and Barnwell formations.

TABLE 3.-Relation of grain size, percent of heavy minerals, and percent of monazite in samples from the Tuscaloosa formation in South Carolina

$\begin{array}{ccccc}\begin{array}{c}\text { Percentage of } \\ \text { sample }\end{array} & \begin{array}{c}\text { Number } \\ \text { of } \\ \text { samples }\end{array} & \begin{array}{c}\text { Heavy minerals in } \\ <0.5 \text { mm fraction } \\ \text { (average percent) }\end{array} & \begin{array}{c}\text { Monazite in } \\ \text { heavy minerals } \\ \text { (average percent) }\end{array} & \begin{array}{c}\text { Monazite in } \\ \text { sediment } \\ \text { (average percent) }\end{array} \\ 15-19 \ldots \ldots & 1 & 1.10 & 2.10 & 0.0035 \\ 20-24 \ldots \ldots & 1 & .26 & 3.40 & .0018 \\ 25-29 \ldots \ldots & 2 & .75 & 4.30 & .0081 \\ 30-34 \ldots \ldots & 6 & .67 & 3.65 & .0074 \\ 35-39 \ldots \ldots & 7 & .74 & 3.84 & .0099 \\ 40-44 \ldots \ldots & 13 & 1.06 & 2.44 & .0103 \\ 45-49 \ldots \ldots & 7 & .65 & 4.20 & .0123 \\ 50-54 \ldots \ldots & 20 & .47 & 2.41 & .0057 \\ 55-59 \ldots \ldots & 11 & .58 & 3.76 & .0120 \\ 60-64 \ldots \ldots & 15 & .50 & 2.33 & .0070 \\ 65-69 \ldots \ldots & 13 & .45 & 3.88 & .0113 \\ 70-74 \ldots \ldots & 12 & .42 & 2.19 & .0064 \\ 75-79 \ldots \ldots & 11 & .58 & 2.57 & .0112 \\ 80-84 \ldots \ldots & 12 & .59 & 3.76 & .0177 \\ 85-89 \ldots \ldots & 11 & .37 & 2.63 & .0083 \\ 90-94 \ldots \ldots & 6 & .20 & 1.70 & .0031 \\ 95-99 \ldots \ldots & 2 & .15 & .5 .50 & .0050 \\ 100 \ldots \ldots & 1 & .40 & .70 & .0028\end{array}$

\section{GEOGRAPHIC DISTRHBUTION OF MONAZITE}

Monazite in the Tuscaloosa formation and other sediments sampled is thought to have been derived from monazite-bearing rocks in the Piedmont that have been shown (Mertie, 1953, pl. 1) to occur principally in an eastern and a western belt (fig. 45). The data shown on plates 19-22 do not suggest derivation from any particular part or parts of these belts, or the direction or means by which sediment was transported from them to the Coastal Plain. However, most of 
the samples have been examined only for the amount of contained monazite; the data do not show whether study of the heavy-mineral suites as a whole, or other studies, would reveal more closely the sources or the ways in which monazite was deposited in the Coastal Plain sediments.

\section{NORTH CAROLINA}

East of a north-south line just east of Fayetteville, N. C., in the northeastern corner of the area covered by plate 19 the monazite content of the sediments is comparatively low, chiefly because of a low percentage of monazite in the heavy minerals. Such low percentages would not be expected if the past drainage had been similar to that of the present and if the eastern monazite belt had been yielding appreciably monazite-bearing sediment to the Coastal Plain.

On the average, monazite content of the sediments is distinctly higher southwest of the eastern monazite belt and southwest from the longitude of Fayetteville. Some of the highest contents are found between that community and the N.C.-S.C. State line. More than 1 pound of monazite per cubic yard is in the three samples numbered 51,62 , and 64 which are from, respectively, pink-to-yellow, silty, fine-grained sand ; silty, pebbly, arkosic sand with local beds of limonitic sandstone; and orange-to-olive, pebbly, silty, fine-grained sand.

\section{SOUTH CAROLINA}

A group of samples of comparatively high monazite content was collected near the northeastern corner of the area in South Carolina (pl. 20). Of these, sample 125, although mapped as Tuscaloosa by Cooke (1936, pl. 2), was judged by the collector to be Pleistocene, because it consisted of loose, yellow, fine-grained sand. About 20 miles west of the Pee Dee River, a large number of samples contain half a pound or more of monazite per cubic yard of sediment; of these, No. 137 is exceptionally rich in monazite. This sample is a red, silty pebbly sand from the base of the Tuscaloosa formation. The Pee Dee River, by way of the Yadkin River, now drains one of the narrow parts of the western monazite belt. Perhaps a more easterly system of drainage existed in Tuscaloosa time and transported monazite from wider parts of the belt around the present boundary between North and South Carolina.

The monazite content is low among sediments in the area between the Pee Dee River and Lynches River; then westward to the Wateree River the content increases in samples 232, 212, 234, 235, and 229. Samples 238 and 271 have exceptionally high percentages of monazite in heavy minerals; sample 238 was the second highest of all samples 
tested. From the Wateree to the Congaree River, values are rather consistently low for both percent of monazite in the heavy minerals and pounds of monazite per cubic yard of sediment.

West of the Congaree is a large area in which there are many samples of high monazite content. More detailed work should be done in this part of South Carolina, because the high monazite concentrations are in three formations: the Tuscaloosa, the McBean, and the Barnwell. The Congaree and its tributaries drain the widest part of the western monazite belt, and high percentages of monazite in Coastal Plain sediments nearby suggest a large source area for monazite and a similar drainage in the past. The McBean and Barnwell formations may have derived their monazite by erosion and redeposition of materials from the Tuscaloosa.

Samples with comparatively little monazite characterize that part of South Carolina between the rich area west of the Congaree River and the State boundary with Georgia. In this large expanse only locality 314 is outstanding for monazite, but a few other places (samples 279, 303, and 313) have about 0.5 pound of monazite per cubic yard. Sediments in this southwestern part of South Carolina contain less monazite than those in any area of comparable size sampled in the present work.

\section{GEORGIA}

Samples (pl. 21) from the Tuscaloosa formation in Georgia average about the same amount of monazite per cubic yard as do samples from the same formation in South Carolina: about 0.3 and 0.25 pound, respectively. Northeast of the Ogeechee River only two samples from the Tuscaloosa formation, Nos. 321 and 325, have more than the average amount of monazite.

The distribution of monazite in Georgia, apart from this northeastern part, has no distinctive pattern. Only two samples, Nos. 377 and 386, contain more than a pound of monazite per cubic yard. No. 377 , from the Tuscaloosa formation, is a pink-to-orange silty sand, with 98 percent passing through the $0.5 \mathrm{~mm}$ sieve. No. 386, with a content of 2.10 pounds of monazite per cubic yard, comes from the base of the Tuscaloosa and is the richest sample taken in the entire area studied. When the sample was collected some of the larger pebbles in the pebbly sand were excluded from the sampling. It is estimated that the monazite content of a more representative sample would be less than 2 pounds but would still be unusually high. Sample 359 , although containing less than a pound of monazite per cubic yard of sediment, has the highest percentage (11.9 percent) of monazite in heavy minerals found during this work. 
ALABAMA

The 17 samples of the Tuscaloosa formation taken in Alabama (pl. 22) have an average monazite content of only 0.1 pound per cubic yard. This relatively low content is due in part to a low percentage of monazite in the heavy minerals. The samples from Alabama average only 2 percent monazite in the heavy minerals, whereas the 151 samples from the Tuscaloosa formation in South Carolina average 3 percent. It is difficult to explain such low content, since all samples lie comparatively close to a wide part of Mertie's (1953) western monazite belt. Further, monazite content decreases westward in Alabama, despite the fact that such samples as Nos. 390, 391, and 392 come from very close to the overlapped end of the monazite belt. A possible explanation for low monazite content in these sediments may be low content in the rocks in the southwestern part of the western monazite belt; or it may be that this part of the belt was covered during Tuscaloosa time, initially, perhaps, by the lowest beds of the Tuscaloosa itself.

\section{GRADE AND SIZE OF PLACERS}

Sediments containing 1 percent or more of heavy minerals possibly might be called placers because they have a concentration of heavy minerals well above the average for Coastal Plain sediments. Of the 456 samples examined, only 25 contained 1 percent or more of heavy minerals. Monazite is generally present as only a small proportion of the heavy minerals; in 151 samples of the Tuscaloosa formation in South Carolina, for example, it averages 3 percent and the two highest concentrations found in the present work are 11.9 and 10.1 percent. In terms of monazite content of rock, the 151 South

- Carolina samples from the Tuscaloosa formation average about 0.25 pound per cubic yard, and the two samples with 11.9 and 10.1 percent of monazite in heavy minerals contain about 0.8 pound per cubic yard.

The location of all samples with a content of 0.25 pound or more is given in detail in table 2. The richest sample examined gave 2.1 pounds per cubic yard; richer samples might be found by additional sampling.

Some of the samples reported here are possibly of commercial or near-commercial grade, but additional field and laboratory work will be necessary to establish reliable figures for tonnage.

The data given on the maps and in the tables pertain only to the samples from the localities indicated. They do not imply that the monazite content shown for two localities is maintained between them, or that the content between them is an average of the two 
values. That values may be roughly constant between two localities is suggested by the data from samples 321 and 322 . The heavy-mineral suite of sample 321 is almost all staurolite. Sample 322, taken about 5 miles away, shows a similar high percentage of staurolite. These two samples are the only ones with this exceptional heavymineral suite, and the implication is that the high content of staurolite may be widespread in this area. By extension, the content of monazite may be widespread too, though there are no specific data to support this assumption.

There are few localities that give information about vertical continuity of the monazite. One of the best is locality 314 (Barnwell formation, South Carolina). Sample 314C was taken 15 feet above 314B; the two have almost identically high percentages of monazite. Another locality where the samples have almost the same percentages of monazite is 377 (McBean formation, Georgia) where sample 337A was taken 20 feet stratigraphically higher than 337B. Sample $337 \mathrm{~A}$ is finer grained than $337 \mathrm{~B}$ and has a somewhat higher percentage of heavy minerals, consequently it has a higher content of monazite. At these two localities and at other places the vertical continuity of monazite content between the samples has not been proved, but there is no reason to assume that such tenors are restricted to the sampled intervals only. However, samples 118, North Carolina, and 128, South Carolina (both from the Tuscaloosa formation), are about 50 feet apart stratigraphically, but their monazite contents are considerably different.

Samples have been collected from sediments of the Pleistocene and the Tuscaloosa formation (nos. 72, 81, 87, 89, 101, 102, 106, and 112) at the same localities, and the two samples of each pair from the same locality are usually quite similar with respect to amount of contained monazite. This suggests that material of Pleistocene age is made up in large part of reworked Tuscaloosa formation.

Promising areas for further work are outlined in plates 19-22 and in the discussion on "Geographic distribution." The formations which unconformably overlie the Tuscaloosa formation and which presumably are made up in considerable part of reworked material from the Tuscaloosa are particularly promising. The 5 samples of the $\mathrm{McBean}$ formation in South Carolina have an average content of 0.7 pound of monazite per cubic yard. These formations probably have their highest monazite contents near the contact with the Tuscaloosa formation, and presumbly, because of their regular bedding, such high content would continue laterally for a considerable distance. 


\section{MONAZITE IN RECENT STREAM SEDIMENTS AND FLOOD PLAINS}

One heavy-mineral suite characterizes nearly all the formations sampled for this report. The only other suite, which contains epidote, hornblende, and garnet in addition to the usual heavy minerals, is

- found in the sediments and flood plains of streams that drain the Piedmont province. The monazite contents of the two suites of heavy minerals are different. Of 10 alluvial deposits sampled in South Carolina along streams that flow from the Piedmont into and across the Coastal Plain, the average suite of heavy minerals contained only 0.25 percent monazite. No monazite was in 6 of the 10 samples.

- Streams that head within the Coastal Plain were sampled at 8 places and have an average of 3.7 percent of monazite in the heavy-mineral fraction. The poorest sample contains 1.3 percent monazite in the heavy fraction.

There are many possible reasons for this difference in monazite content. One may be the method of collecting. Most of the streams that drain the Piedmont province are large, are bordered by swamp, and are difficult to sample. At many places samples from these streams represent sediments deposited during overbank floods and might not contain monazite. However, this explanation seems inadequate, since samples from streams rising in the Piedmont actually contain a higher proportion of heavy minerals than samples from streams that flow only in the Coastal Plain. The ratio is 2.7 percent to 1.3 percent. Another possibility is that some monazite-poor streams from the Piedmont do not reach and drain monazite-bearing rocks. Of the 10 samples examined, however, 6 came from rivers forming the WatereeCongaree system, which drains the widest part of the western monazite belt. Whatever the explanation for lack of monazite, it is clear that the larger streams flowing from the Piedmont are not receiving a high proportion of their sediment from the Tuscaloosa and other monazite-bearing formations of the Coastal Plain.

\section{OTHER MINERAI PRODUCTS}

Other minerals and materials associated with monazite may be $\rightarrow$ salable as coproducts or byproducts under favorable circumstances. The most salable commodities are sand and gravel, titanium-bearing minerals, zircon, and high-alumina minerals.

Sand and gravel are widely quarried in operations that range in size from small pits supplying road material for local use to quarries reported to produce several thousand tons a day. None of the samples showing highest percentages of monazite in the heavy minerals came from quarries, but a high content of monazite might make it possible 
to work ground for sand, gravel, and monazite where it is currently uneconomical to mine for sand and gravel alone.

The titanium-bearing minerals ilmenite, leucoxene, and rutile are the most important industrial minerals in the sediments. Estimates were made of the quantity of titanium-bearing minerals in the heavy fractions of 12 samples from the Tuscaloosa formation (nos. 51, 62, $64,137,157,170,193 \mathrm{~B}, 334 \mathrm{~A}, 350,359,368$, and 386) in North Carolina, South Carolina, and Georgia. The samples were chosen because they generally have a higher than average percent of monazite. The percent of opaques (dominantly ilmenite and leucoxene) varied from 41 to 78 and averaged 55.5. Rutile made up 2.3 to 11.7 percent of the heavy minerals and averaged 5.1 percent. No determinations of titanium dioxide were made in this study.

Zircon averaged 20.5 percent of the heavy fraction in the same 12 samples and ranged in amount from 9.0 percent to 32.6 percent.

The high-alumina minerals include kyanite, sillimanite, and staurolite, and all range widely in their percent distribution in these 12 samples. Staurolite made up 0.3 to 13.7 percent of the heavy minerals and averaged 2.8 percent. Kyanite and sillimanite together form less than 2 percent of the heavy minerals in 8 samples, but are 5.5, 10.1, 9.5, and 7.5 percent of the heavy fraction in the other samples.

A sample from the Barnwell formation in South Carolina and a sample from sediments of Pleistocene age in North Carolina show the same minerals in percentages not much different from those $\alpha$. given for the 12 from the Tuscaloosa formation.

Deposits whose value will depend on several coproducts appear to be the only source of monazite along the inner belt of the Coastal Plain. Commercially profitable deposits economically dependent only on monazite will not be found there.

\section{LITERATURE CITED}

Berry, E. W., 1949, Geologic map, Coastal Plain of North Carolina: N. C. Div. Mineral Res.

Cooke, C. W., 1926, The Cenozoic formations, in Geology of Alabama: Ala. Geol. Survey Special Rept. no. 14, p. 251-297.

- 1936, Geology of the Coastal Plain of South Carolina: U. S. Geol. Survey Bull. 867.

- 1943, Geology of the Coastal Plain of Georgia: U. S. Geol. Survey Bull. 941.

Hansen, L. A., and Cuppels, N. P., 1954, Monazite placer on the First Broad River and its tributaries, Cleveland County, N. C.: U. S. Bur. Mines and U. S. Geol. Survey RME-3116, issued by U. S. Atomic Energy Comm. Tech. Inf. Service, Oak Ridge, Tenn. 
1955, Monazite placer at the junction of the North Tyger River with the Middle Tyger River, Spartanburg County, S. C.: U. S. Bur. Mines and U. S. Geol. Survey. RME-3117, issued by U. S. Atomic Energy Comm. Tech. Inf. Service, Oak Ridge, Tenn.

Hansen, L. A., and White, A. M., 1954, Monazite placers on South Muddy Creek, McDowell County, and Silver Creek, Burke County, N. C.: U. S. Bur. Mines and U. S. Geol. Survey RME-3115, issued by U. S. Atomic Energy Comm. Tech. Inf. Service, Oak Ridge, Tenn.

Mertie, J. B., Jr., 1953, Monazite deposits of the southeastern Atlantic States: U. S. Geol. Survey Circ. 237.

Stephenson, L. W., 1912, The Cretaceous, Lafayette, and Quaternary formations, in The Coastal Plain of North Carolina: N. C. Geol. and Econ. Survey, v. 3, p. 73-171, 258-290.

1926, The Mesozoic rocks, in Geology of Alabama: Ala. Geol. Survey Special Rept. no. 14, p. 231-250. 



\section{N D E X}

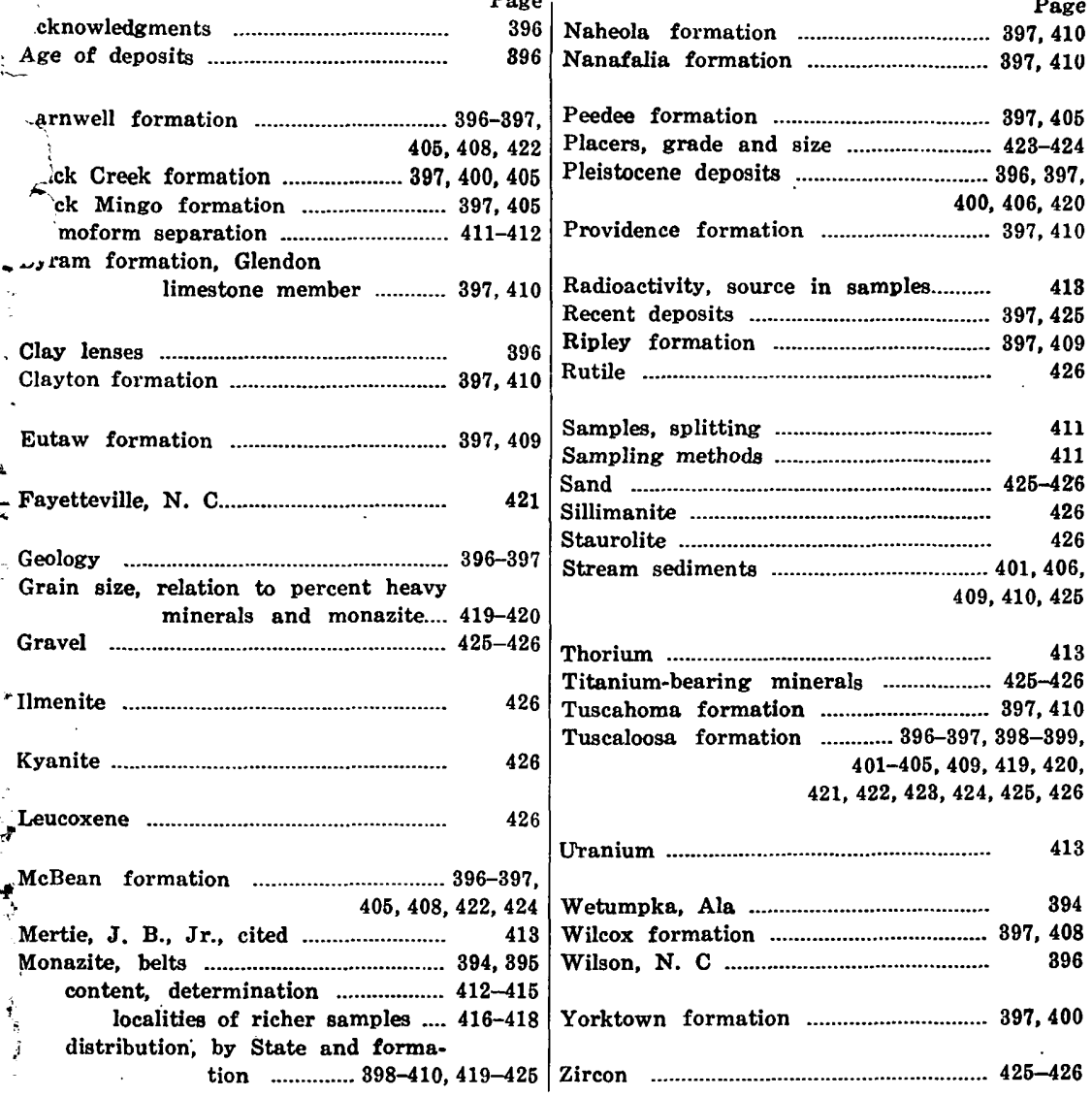

U. S. GOVERNMENT PRINTING OFFICE: $1958-433209$ 